\title{
ABSENCE OF POSITIVE EIGENVALUES FOR HARD-CORE $N$-BODY SYSTEMS
}

\author{
K. ITO AND E. SKIBSTED
}

\begin{abstract}
We show absence of positive eigenvalues for generalized 2-body hardcore Schrödinger operators under the condition of bounded strictly convex obstacles. A scheme for showing absence of positive eigenvalues for generalized $N$-body hard-core Schrödinger operators, $N \geq 2$, is presented. This scheme involves high energy resolvent estimates, and for $N=2$ it is implemented by a Mourre commutator type method. A particular example is the Helium atom with the assumption of infinite mass and finite extent nucleus.
\end{abstract}

\section{CONTENTS}

1. Introduction and results 1

1.1. Usual generalized $N$-body systems 2

1.2. Generalized $N$-body hard-core systems 4

1.3. Geometric properties 6

2. Reduction to high-energy hard-core sub-system resolvent bounds $\quad 7$

2.1. General scheme 8

3. High-energy hard-core one-body resolvent bound 15

$\begin{array}{ll}\text { 3.1. Abstract Besov spaces } & 15\end{array}$

$\begin{array}{ll}\text { 3.2. Setting of problem } & 17\end{array}$

3.3. Besov space bound of resolvent, Lemma 3.5 19

3.4. Besov space bound of resolvent, Proposition $3.6 \quad 25$

3.5. Case $\Omega=\mathbf{X} \quad 26$

Appendix A. 27

A.1. Setting 28

$\begin{array}{ll}\text { A.2. Preliminaries } & 29\end{array}$

A.3. Undoing commutators 31

Appendix B. 33

$\begin{array}{ll}\text { References } & 36\end{array}$

\section{IntRoduction AND RESUlts}

Consider the $N$-body Schrödinger operator

$$
H=\sum_{j=1}^{N}\left(-\frac{1}{2 m_{j}} \Delta_{x_{j}}+V_{j}^{\mathrm{ncl}}\left(x_{j}\right)\right)+\sum_{1 \leq i<j \leq N} V_{i j}^{\text {elec }}\left(x_{i}-x_{j}\right)
$$

for a system of $N d$-dimensional particles in the exterior of a bounded strictly convex obstacle $\Theta_{1} \subset \mathbb{R}^{d}$ (for $N=1$ the last term is omitted). Whence $H$ is an operator on the Hilbert space $L^{2}(\Omega) ; \Omega=\left(\Omega_{1}\right)^{N}, \Omega_{1}=\mathbb{R}^{d} \backslash \bar{\Theta}_{1}$. It is defined more precisely by imposing the Dirichlet boundary condition. This operator models a system of 
$N d$-dimensional charged particles interacting with a fixed nucleus of finite extent, for example a ball (or possibly a somewhat deformed ball). In particular (assuming $0 \in \Theta_{1}$ ) we could have Coulomb interactions $V_{j}^{\text {ncl }}(y)=q_{j} q^{\text {ncl }}|y|^{-1}$ and $V_{i j}^{\text {elec }}(y)=$ $q_{i} q_{j}|y|^{-1}$ in dimension $d \geq 2$. We address the problem of proving absence of positive eigenvalues. While this property is well-known for the one-body problem it is open for $N \geq 2$. We introduce for obstacle problems of this type a general procedure involving high energy resolvent estimates for effective sub-Hamiltonians. We show that this scheme can be implemented for the case $N=2$. In this case essentially such an effective sub-Hamiltonian is a one-body Hamiltonian for an exterior region. The result is shown in the so-called generalized 2-body hard-core framework.

1.1. Usual generalized $N$-body systems. We will work in a generalized framework. We first review the analogue of this without obstacles, i.e. with "soft potentials". This is given by real finite dimensional vector space $\mathbf{X}$ with an inner product $q$, i.e. $(\mathbf{X}, q)$ is Euclidean space, and a finite family of subspaces $\left\{\mathbf{X}_{a} \mid a \in \mathcal{A}\right\}$ closed with respect to intersection. We refer to the elements of $\mathcal{A}$ as cluster decompositions (not to be motivated here). The orthogonal complement of $\mathbf{X}_{a}$ in $\mathbf{X}$ is denoted $\mathbf{X}^{a}$, and correspondingly we decompose $x=x^{a} \oplus x_{a} \in \mathbf{X}^{a} \oplus \mathbf{X}_{a}$. We order $\mathcal{A}$ by writing $a_{1} \subset a_{2}$ if $\mathbf{X}^{a_{1}} \subset \mathbf{X}^{a_{2}}$. It is assumed that there exist $a_{\min }, a_{\max } \in \mathcal{A}$ such that $\mathbf{X}^{a_{\min }}=\{0\}$ and $\mathbf{X}^{a_{\max }}=\mathbf{X}$. Let $\mathcal{B}=\mathcal{A} \backslash\left\{a_{\min }\right\}$. The length of a chain of cluster decompositions $a_{1} \subsetneq \cdots \subsetneq a_{k}$ is the number $k$. Such a chain is said to connect $a=a_{1}$ and $b=a_{k}$. The maximal length of all chains connecting a given $a \in \mathcal{A} \backslash\left\{a_{\max }\right\}$ and $a_{\max }$ is denoted by $\# a$. We define $\# a_{\max }=1$ and denoting $\# a_{\min }=N+1$ we say the family $\left\{\mathbf{X}^{a} \mid a \in \mathcal{A}\right\}$ is of $N$-body type. Whence the generalized 2-body framework is characterized by the condition $\mathbf{X}_{a} \cap \mathbf{X}_{b}=0$ for $a, b \neq a_{\min }, a \neq b$.

The $N$-body Schrödinger operator $H$ introduced above (now considered without an obstacle) can be written on the form

$$
H=H_{0}+V
$$

where $2 H_{0}$ is (minus) the Laplace-Beltrami operator on the space

$$
\mathbf{X}=\left(\mathbb{R}^{d}\right)^{N}, \quad q=\sum_{j=1}^{N} m_{j}\left|x_{j}\right|^{2},
$$

$V=V(x)=\sum_{b \in \mathcal{B}} V_{b}\left(x^{b}\right)$ and indeed the relevant family $\left\{\mathrm{X}^{a} \mid a \in \mathcal{A}\right\}$ of subspaces as discussed above is of $N$-body type. However this is just one example of a generalized $N$-body Schrödinger operator. The general construction of such an operator $H$ is similar, and under the following condition it is well-defined with form domain given by the Sobolev space $H^{1}(\mathbf{X})$, cf. [RS, Theorem X.17].

Condition 1.1. There exists $\varepsilon>0$ such that for potential $V_{b}, b \in \mathcal{B}$, there is a splitting $V_{b}=V_{b}^{(1)}+V_{b}^{(2)}$, where

(1) $V_{b}^{(1)}$ is smooth and

$$
\partial_{y}^{\alpha} V_{b}^{(1)}(y)=O\left(|y|^{-\varepsilon-|\alpha|}\right) .
$$

(2) $V_{b}^{(2)}$ is compactly supported and

$$
(-\Delta+1)^{-1 / 2} V_{b}^{(2)}(-\Delta+1)^{-1 / 2} \text { is compact on } L^{2}\left(\mathbb{R}_{y}^{\operatorname{dim} \mathbf{X}^{b}}\right) .
$$


Let $-\Delta^{a}=\left(p^{a}\right)^{2}$ and $-\Delta_{a}=p_{a}^{2}$ denote (minus) the Laplacians on $L^{2}\left(\mathbf{X}^{a}\right)$ and $L^{2}\left(\mathbf{X}_{a}\right)$, respectively. Here $p^{a}=\pi^{a} p$ and $p_{a}=\pi_{a} p$ denote the internal (i.e. within clusters) and the inter-cluster components of the momentum operator $p=-\mathrm{i} \nabla$, respectively. For $a \in \mathcal{B}$, denote

$$
\begin{aligned}
V^{a}\left(x^{a}\right) & =\sum_{b \subset a} V_{b}\left(x^{b}\right), \\
H^{a} & =-\frac{1}{2} \Delta^{a}+V^{a}\left(x^{a}\right), \\
H_{a} & =H^{a}-\frac{1}{2} \Delta_{a}, \\
I_{a}(x) & =\sum_{b \not \subset a} V_{b}\left(x^{b}\right) .
\end{aligned}
$$

We define $H^{a_{\min }}=0$ on $L^{2}\left(\mathbf{X}^{a_{\min }}\right):=\mathbb{C}$. The operator $H^{a}$ is the sub-Hamiltonian associated with the cluster decomposition $a$ and $I_{a}$ is the sum of all inter-cluster interactions. The detailed expression of $H^{a}$ depends on the choice of coordinates on $\mathrm{X}^{a}$.

In a natural way we have sub-Hamiltonians $H^{a}$ and "inter-cluster" Hamiltonians $H_{a}=H^{a} \otimes I+I \otimes \frac{1}{2} p_{a}^{2}$. Given a family $\left\{\mathrm{X}^{a} \mid a \in \mathcal{A}\right\}$ of $N$-body type and imposing Condition 1.1 the generalized $N$-body Hamiltonian is $H=H^{a_{\max }}$.

Let

$$
\mathcal{T}=\cup_{a \in \mathcal{A}, \# a \geq 2} \sigma_{\mathrm{pp}}\left(H^{a}\right)
$$

be the set of thresholds of $H$. The HVZ theorem [RS, Theorem XIII.17] gives the bottom of the essential spectrum $\Sigma_{2}:=\inf \sigma_{\text {ess }}(H)$ of $H$ by the formula

$$
\Sigma_{2}=\min _{a \in \mathcal{A} \backslash\left\{a_{\max }\right\}} \inf \sigma\left(H^{a}\right)=\min _{a \in \mathcal{A}, \# a=2} \inf \sigma\left(H^{a}\right) .
$$

It is also well-known that under rather general conditions $H$ does not have positive eigenvalues and the negative eigenvalues can at most accumulate at the thresholds from below, see $[\mathrm{FH}]$ and $[\mathrm{Pe}]$.

1.1.1. Graf vector field. We give a brief review of the construction of a family of conjugate operators for $N$-body Hamiltoninans originating from [Sk1]. A slightly different proof appears in [Sk2]. This construction is based on the vector field invented by Graf [Gra] which is a vector field satisfying the following properties, cf. $\left[\mathrm{Sk} 2\right.$, Lemma 4.3]. We use throughout the paper the notation $\langle x\rangle=\sqrt{x^{2}+1}$ and $\mathbb{N}_{0}=\mathbb{N} \cup\{0\}$.

Lemma 1.2. There exist on $\mathbf{X}$ a smooth vector field $\omega$ with symmetric derivative $\omega_{*}$ and a partition of unity $\left\{\tilde{q}_{a}\right\}$ indexed by $a \in \mathcal{A}$ and consisting of smooth functions, $0 \leq \tilde{q}_{a} \leq 1$, such that for some positive constants $r_{1}$ and $r_{2}$

(1) $\omega_{*}(x) \geq \sum_{a} \pi_{a} \tilde{q}_{a}$.

(2) $\omega^{a}(x)=0$ if $\left|x^{a}\right|<r_{1}$.

(3) $\left|x^{b}\right|>r_{1}$ on $\operatorname{supp}\left(\tilde{q}_{a}\right)$ if $b \not \subset a$.

(4) $\left|x^{a}\right|<r_{2}$ on $\operatorname{supp}\left(\tilde{q}_{a}\right)$.

(5) For all $\alpha \in \mathbb{N}_{0}^{\operatorname{dim} \mathbf{X}}$ and $k \in \mathbb{N}_{0}$ there exist $C \in \mathbb{R}$ :

$$
\left|\partial_{x}^{\alpha} \tilde{q}_{a}\right|+\left|\partial_{x}^{\alpha}(x \cdot \nabla)^{k}(\omega(x)-x)\right| \leq C .
$$

Now, proceeding as in $[\mathrm{Sk} 2]$, we introduce the rescaled vector field $\omega_{R}(x):=R \omega\left(\frac{x}{R}\right)$ and the corresponding operator

$$
A=A_{R}=\omega_{R}(x) \cdot p+p \cdot \omega_{R}(x) ; R>1 .
$$


We also introduce the function $d: \mathbb{R} \rightarrow \mathbb{R}$ by

$$
d(E)=\left\{\begin{array}{l}
\left.\left.\inf _{\tau \in \mathcal{T}(E)}(E-\tau), \mathcal{T}(E):=\mathcal{T} \cap\right]-\infty, E\right] \neq \emptyset \\
1, \mathcal{T}(E)=\emptyset
\end{array}\right.
$$

These devices enter into the following Mourre estimate. We remark that all inputs needed for the proof are stated in Lemma 1.2 and that although [Sk2, Corollary 4.5] is stated for relatively operator compact potentials the proof of [Sk2] generalizes to include the class of relatively form compact potentials of Condition 1.1. For a different proof we refer to [Gri].

Lemma 1.3. For all $E \in \mathbb{R}$ and $\epsilon>0$ the exists $R_{0}>1$ such that for all $R \geq R_{0}$ there is a neighbourhood $\mathcal{V}$ of $E$ and a compact operator $K$ on $L^{2}(\mathbf{X})$ such that

$$
f(H)^{*} \mathrm{i}\left[H, A_{R}\right] f(H) \geq f(H)^{*}\{4 d(E)-\epsilon-K\} f(H) \text { for all } f \in C_{\mathrm{c}}^{\infty}(\mathcal{V}) .
$$

Here the commutator is given by (1.13) stated below. The possibly existing local singularities of the potential does not enter (for $R$ large) due to Lemma 1.2 (2). This feature motivates application to hard-core models, see Subsection 1.2.

Two of the consequences of a Mourre estimate like the one stated above are that the set of thresholds $\mathcal{T}$ is closed and countable and that the eigenvalues of $H$ can at most accumulate at $\mathcal{T}$. We discuss a third consequence, decay of non-threshold eigenstates, in Subsection 1.2.

1.2. Generalized $N$-body hard-core systems. The generalized hard-core model is a modification for the above model. For the generalized hard-core model we are given for each $a \in \mathcal{B}$ an open subset $\Omega_{a} \subset \mathbf{X}^{a}$ with $\mathbf{X}^{a} \backslash \Omega_{a}$ compact, possibly $\Omega_{a}=\mathbf{X}^{a}$. Let for $a_{\min } \neq b \subset a$

$$
\Omega_{b}^{a}=\left(\Omega_{b}+\mathbf{X}_{b}\right) \cap \mathbf{X}^{a}=\Omega_{b}+\mathbf{X}_{b} \cap \mathbf{X}^{a},
$$

and for $a \neq a_{\text {min }}$

We define $\Omega^{a_{\min }}=\{0\}$.

$$
\Omega^{a}=\cap_{a_{\min } \neq b \subset a} \Omega_{b}^{a} .
$$

Condition 1.4. There exists $\varepsilon>0$ such that for all $b \in \mathcal{B}$ there is a splitting $V_{b}=V_{b}^{(1)}+V_{b}^{(2)}$, where

(1) $V_{b}^{(1)}$ is smooth on the closure of $\Omega_{b}$ and

$$
\partial_{y}^{\alpha} V_{b}^{(1)}(y)=O\left(|y|^{-\varepsilon-|\alpha|}\right) .
$$

(2) $V_{b}^{(2)}$ vanishes outside a bounded set in $\Omega^{b}$ and

$$
V_{b}^{(2)} \in \mathcal{C}\left(H_{0}^{1}\left(\Omega_{b}\right), H_{0}^{1}\left(\Omega_{b}\right)^{*}\right) .
$$

Here and henceforth, given Banach spaces $X_{1}$ and $X_{2}$, the notation $\mathcal{C}\left(X_{1}, X_{2}\right)$ and $\mathcal{B}\left(X_{1}, X_{2}\right)$ refers to the set of compact and the set of bounded operators $T$ : $X_{1} \rightarrow X_{2}$, respectively.

We consider for $a \in \mathcal{B}$ the Hamiltonian $H^{a}=-\frac{1}{2} \Delta_{x^{a}}+V^{a}$ on $L^{2}\left(\Omega^{a}\right)$ with Dirichlet boundary condition on $\partial \Omega^{a}$, in particular $H=\frac{1}{2} p^{2}+V$ on $L^{2}(\Omega)$ with Dirichlet boundary condition on $\partial \Omega$ where $\Omega:=\Omega^{a_{\max }}$. The corresponding form domain is the Sobolev space $H_{0}^{1}\left(\Omega^{a}\right)$. Due to the continuous embedding $H_{0}^{1}\left(\Omega^{a}\right) \subset H_{0}^{1}\left(\Omega_{b}^{a}\right)$ for $a_{\text {min }} \neq b \subset a$ we conclude that indeed $H^{a}$ is self-adjoint, cf. [RS, Theorem X.17]. Again we define $H^{a_{\min }}=0$, and the set of thresholds is also given as in 
Subsection 1.1. We note that one can replace the Hilbert space $L^{2}(\mathbf{X})$ in Lemma 1.3 by $\mathcal{H}:=L^{2}(\Omega)$ and then obtain a Mourre estimate for the present Hamiltonian $H$, cf. [Gri, Theorem 2.4]. All what is needed for this is to make sure that $R>1$ is so large that the rescaled Graf vector field $\omega_{R}$ either vanishes or acts tangentially on the boundary $\partial \Omega$. The latter is doable due to Lemma $1.2(2)$.

According to [Gri, Theorem 2.5(1)] non-threshold eigenstates decay exponentially at rates determined by thresholds above the corresponding eigenvalues. This is a consequence of the hard-core Mourre estimate by arguments similar to the ones of $[\mathrm{FH}]$ for usual $N$-body Hamiltonians. In [Gri] Griesemer states as an open problem absence of positive eigenvalues under an additional connectedness condition. This is the problem we shall address in the present paper. The pattern of proof of $[\mathrm{FH}]$ does not work except the following induction scheme: For $N=1$ absence of positive eigenvalues follows from various papers (assuming that $\Omega \subset \mathbf{X}$ is connected), for example most recently [IS2]. For $N \geq 2$ we could suppose by induction that the result holds for sub-Hamiltonians, whence that there are no positive thresholds. Using the hard-core Mourre estimate in a similar way as for soft potentials [FH, Gri, IS2] we then deduce that an eigenstate with corresponding positive eigenvalue would decay super-exponentially, cf. [Gri, Theorem 2.5(1)]. This would be derived in terms of the potential function $r$ discussed below. Whence for any such eigenstate $\phi$ (i.e. corresponding to a positive eigenvalue) we would have $\mathrm{e}^{\sigma r} \phi \in L^{2}(\Omega)$ for all $\sigma \geq 0$. Consequently what would remain to be shown for completing the induction argument is that super-exponentially decaying eigenstates vanish.

Although we are not able in general to implement the above scheme for showing absence of positive eigenvalues we show a partial result which reduces the problem to resolvent estimates for sub-system type Hamiltonians. Moreover we do in fact implement the scheme for $N=2$ under additional conditions.

Condition 1.5. Suppose $N \geq 2$. For all $b \in \mathcal{B} \backslash\left\{a_{\max }\right\}$ with $\Omega_{b} \subsetneq \mathbf{X}^{b}$ the set $\Theta_{b}:=\mathbf{X}^{b} \backslash \overline{\Omega_{b}} \neq \emptyset$ has smooth boundary $\partial \Theta_{b}=\partial \Omega_{b}$ and is strictly convex.

For the notion of strict convexity used in this paper we refer to Appendix B. Given Condition 1.5, by definition if $\Omega_{b} \subsetneq \mathbf{X}^{b}$, then $\operatorname{dim} \mathbf{X}^{b} \geq 2$. With minor modifications we could have allowed $\operatorname{dim} \mathbf{X}^{b}=1$ in the definition of strict convexity and obtained the same results, however for convenience we prefer not to do that.

The main result of this paper is the following.

Theorem 1.6. Suppose $N=2$ and Conditions 1.4 and 1.5. Suppose that for all $b \in \mathcal{B} \backslash\left\{a_{\max }\right\}$ with $\Omega_{b} \subsetneq \mathbf{X}^{b}$ the term $V_{b}^{(2)}=0$ while for all $b \in \mathcal{B} \backslash\left\{a_{\max }\right\}$ with $\Omega_{b}=\mathbf{X}^{b}$

$$
x^{b} \cdot \nabla V_{b}^{(2)}\left(x^{b}\right),\left(x^{b} \cdot \nabla\right)^{2} V_{b}^{(2)}\left(x^{b}\right) \in \mathcal{C}\left(H^{1}\left(\mathbf{X}^{b}\right), H^{1}\left(\mathbf{X}^{b}\right)^{*}\right) .
$$

Suppose that any eigenstate of $H$ vanishing at infinity must be zero (the unique continuation property). Then $H$ does not have positive eigenvalues.

The unique continuation property is a well-studied subject, see for example [Ge, JK, RS, Wo]. It is valid for a large class of potential singularities given connectedness of $\Omega$.

Corollary 1.7. For $N=2$ charged particles confined to the exterior of a bounded strictly convex obstacle $\Theta_{1} \subset \mathbb{R}^{d}$ containing $0, d \geq 2$, the corresponding Hamiltonian $H$ given by (1.1) with Coulomb interactions $V_{j}^{\mathrm{ncl}}(y)=q_{j} q^{\mathrm{ncl}}|y|^{-1}$ and $V_{i j}^{\text {elec }}(y)=$ $q_{i} q_{j}|y|^{-1}$ does not have positive eigenvalues. 
Note for Corollary 1.7 that indeed $\Omega=\left(\Omega_{1}\right)^{2} \backslash\left\{\left(x_{1}, x_{2}\right) \in\left(\mathbb{R}^{d}\right)^{2} \mid x_{1}=x_{2}\right\}$ is a connected subset of $\mathbb{R}^{2 d}$ for $d \geq 2$ (which follows readily using that $\Omega_{1} \subset \mathbb{R}^{d}$ is connected) and that the version of the unique continuation property of [RS] applies.

Another result of this paper is the following statement in which a technical condition stated in Section 2 enters.

Proposition 1.8. Suppose $N \geq 2$ and Conditions 1.4 and 2.1. Suppose $H$ does not have positive thresholds. Suppose that any eigenstate of $H$ vanishing at infinity must be zero (the unique continuation property). Then $H$ does not have positive eigenvalues.

By imposing the analogous version of Condition 2.1 for sub-Hamiltonians as well as the unique continuation property for these operators and for $H$ (in addition to Condition 1.4) we obtain that $H$ does not have thresholds nor positive eigenvalues, cf. the scheme discussed above. However since we are only able to verify Condition 2.1 for $N=2$ using Condition 1.5 we need these restrictions in Theorem 1.6. Nevertheless, since proving Condition 2.1 for higher $N$ under Condition 1.4 possibly as well as under Condition 1.5 could be a purely technical difficulty, we consider the statement of Proposition 1.8 in such situation as a result of independent interest. We devote Section 2 to the crucial step in the proof. Section 3 is devoted to the verification of Condition 2.1 for $N=2$. Supplementary material is given in Appendices $\mathrm{A}$ and $\mathrm{B}$.

1.3. Geometric properties. We complete this section by a brief discussion of some properties related to Lemma 1.2, and we show an estimate which may be viewed as a first step in a proof of (a hard-core version of) Lemma 1.3 (not to be elaborated on in this paper). These properties will be important in Section 2.

\subsubsection{Potential function. Since $\omega_{*}$ is symmetric we can write}

$$
\omega=\nabla r^{2} / 2 \text {. }
$$

It will be important for us that the function $r=r(x)$ can be chosen positive, smooth and convex, see the proof of [De, Proposition 4.4] (we remark that [De] also uses the Graf construction although with a different regularization procedure). From the convexity of $r$ we learn that

$$
\partial^{r}|\mathrm{~d} r|^{2} \geq 0 ; \partial^{r} f=\mathrm{i} p^{r} f:=\nabla r \cdot \nabla f .
$$

We have a slight extension of part of (1.5), cf. [De, Lemma $4.3 \mathrm{f})$ ],

$$
\forall \alpha \in \mathbb{N}_{0}^{\operatorname{dim} \mathbf{X}} \text { and } k \in \mathbb{N}_{0}:\left|\partial_{x}^{\alpha}(x \cdot \nabla)^{k}\left(r^{2}-x^{2}\right)\right| \leq C_{\alpha} .
$$

In particular we obtain yet another useful property

$$
\forall \alpha \in \mathbb{N}_{0}^{\operatorname{dim} \mathbf{X}}:\left|\partial_{x}^{\alpha}\left(|\mathrm{d} r|^{2}-1\right)\right| \leq C_{\alpha}\langle x\rangle^{-2} .
$$

In fact letting $f=r^{2}-x^{2}$ the bounds (1.11c) follow from (1.11b) and the identity

$$
|\mathrm{d} r|^{2}-1=\frac{x \cdot \nabla f+4^{-1}|\mathrm{~d} f|^{2}-f}{x^{2}+f} .
$$

The rescaled $r$ reads

$$
r_{R}(x)=\operatorname{Rr}(x / R),
$$

so that $\omega_{R}=\nabla r_{R}^{2} / 2$. Clearly the bounds (1.11a)-(1.11c) are also valid for the rescaled $r$ (possibly with $R$-dependent constants). We also rescale the partition 
functions of Lemma $1.2 \tilde{q}_{a, R}(x):=\tilde{q}_{a}(x / R)$ and similarly for the "quadratic" partition functions

$$
q_{b}(x)=\tilde{q}_{b}(k x)\left(\sum_{c} \tilde{q}_{c}(k x)^{2}\right)^{-1 / 2} ; k=r_{1} / r_{2} .
$$

Using that

$$
\tilde{q}_{c}(x) \tilde{q}_{b}(k x)=0 \text { if } c \not \subset b,
$$

and Lemma 1.2 (1) we conclude that

$$
\omega_{*}(x) \geq \sum_{b} \pi_{b} q_{b}^{2}(x)
$$

1.3.2. Commutator calculation. We calculate

$$
\mathrm{i}\left[H, A_{R}\right]=2 p \omega_{*}(x / R) p-\left(4 R^{2}\right)^{-1}\left(\Delta^{2} r^{2}\right)(x / R)-2 \omega_{R} \cdot \nabla V,
$$

and using (1.12) we thus deduce

$$
\begin{aligned}
\mathrm{i}\left[H, A_{R}\right] & \geq 2 \sum_{b} q_{b, R} p_{b}^{2} q_{b, R}+O\left(R^{-2}\right)-2 \omega_{R} \cdot \nabla V \\
& =2 \sum_{b} q_{b, R} p_{b}^{2} q_{b, R}+O\left(R^{-\min \{2, \varepsilon\}}\right) .
\end{aligned}
$$

1.3.3. More notation. We fix a non-negative $\chi \in C^{\infty}(\mathbb{R})$ with $0 \leq \chi \leq 1$ and

$$
\chi(t)= \begin{cases}0 & \text { for } t \leq 5 / 4 \\ 1 & \text { for } t \geq 7 / 4\end{cases}
$$

We shall frequently use the rescaled function

$$
\chi_{\nu}(t)=\chi(t / \nu), \quad \nu>0
$$

and the notation $\chi_{\nu}^{+}=\chi_{\nu}$ and $\chi_{\nu}^{-}=\bar{\chi}_{\nu}=1-\chi_{\nu}$.

For any self-adjoint operator $T$ and state $\phi$ we write $\langle T\rangle_{\phi}=\langle\phi, T \phi\rangle$ for the corresponding expectation value.

\section{REDUCTION TO HIGH-ENERGY HARD-CORE SUB-SYSTEM RESOLVENT BOUNDS}

Under Condition 1.4 we propose a scheme for showing

$$
(H-E) \phi=0, E>0, \text { and } \forall \sigma \geq 0: \mathrm{e}^{\sigma r} \phi \in \mathcal{H}=L^{2}(\Omega) \Rightarrow \phi=0 .
$$

Here and henceforth $r=r_{R}$ is the rescaled potential function. We suppress the dependence on $R$ which is fixed (large) from this point. The proposed method relies on the unique continuation property and certain high-energy hard-core sub-system type resolvent bounds. The latter are stated in Condition 2.1 given below. Whence we give the crucial step of the proof Proposition 1.8. 
2.1. General scheme. For $\phi$ given as in (2.1) we let for any $\nu \geq 1$ and $\sigma \geq 0$

$$
\phi_{\sigma}=\phi_{\sigma, \nu}:=\chi_{\nu} \mathrm{e}^{\sigma(r-4 \nu)} \phi \in \mathcal{H} ; \chi_{\nu}=\chi_{\nu}(r) \text {. }
$$

Putting $H^{\sigma}=H-\frac{\sigma^{2}}{2}|\mathrm{~d} r|^{2}$ we note that

$$
\left(H^{\sigma}-E\right) \phi_{\sigma}=-\mathrm{i} \sigma\left(\operatorname{Re} p^{r}\right) \phi_{\sigma}-\mathrm{ie}^{\sigma(r-4 \nu)} R(\nu) \phi,
$$

where $R(\nu)=\mathrm{i}\left[H_{0}, \chi_{\nu}\right]=\operatorname{Re}\left(\chi_{\nu}^{\prime} p^{r}\right)$. Whence by undoing the commutator, cf. Appendix A,

$$
\left\langle\mathrm{i}\left[H^{\sigma}, A\right]\right\rangle_{\phi_{\sigma}}=-2 \sigma \operatorname{Re}\left\langle\left(\operatorname{Re} p^{r}\right) A\right\rangle_{\phi_{\sigma}}-2 \operatorname{Re}\left\langle R(\nu) \mathrm{e}^{\sigma(r-4 \nu)} A \chi_{\nu} \mathrm{e}^{\sigma(r-4 \nu)}\right\rangle_{\phi} .
$$

The first term of (2.4) is computed

$$
\begin{aligned}
& -2 \sigma \operatorname{Re}\left(\left(\operatorname{Re} p^{r}\right) A\right) \\
& =-\sigma\left(\operatorname{Re} p^{r}\right)\left(2 r \operatorname{Re} p^{r}-\mathrm{i}|\mathrm{d} r|^{2}\right)+\text { h.c. } \\
& =-4 \sigma\left(\operatorname{Re} p^{r}\right) r \operatorname{Re} p^{r}+\sigma\left(\partial^{r}|\mathrm{~d} r|^{2}\right) .
\end{aligned}
$$

As for the second term we estimate (recall the notation $\bar{\chi}_{\nu}=1-\chi_{\nu}$ )

$$
\begin{aligned}
- & 2 \operatorname{Re}\left\langle R(\nu) \mathrm{e}^{\sigma(r-4 \nu)} A \chi_{\nu} \mathrm{e}^{\sigma(r-4 \nu)}\right\rangle_{\phi} \\
\leq & \left\|\mathrm{e}^{\sigma(r-4 \nu)} R(\nu) \phi\right\|^{2}+\left\|\bar{\chi}_{2 \nu} A \chi_{\nu} \mathrm{e}^{\sigma(r-4 \nu)} \phi\right\|^{2} \\
\leq & \left\{\left\|\chi_{\nu}^{\prime} \mathrm{e}^{\sigma(r-4 \nu)} p^{r} \phi\right\|+\frac{1}{2}\left\|\left(\chi_{\nu}^{\prime \prime}|\mathrm{d} r|^{2}+\chi_{\nu}^{\prime}(\Delta r)\right) \mathrm{e}^{\sigma(r-4 \nu)} \phi\right\|\right\}^{2} \\
& +\left\{\left\|2 r \bar{\chi}_{2 \nu} \chi_{\nu} \mathrm{e}^{\sigma(r-4 \nu)} p^{r} \phi\right\|+\left\|\bar{\chi}_{2 \nu}\left(2 r|\mathrm{~d} r|^{2} \chi_{\nu}^{\prime}+2 \sigma r \chi_{\nu}|\mathrm{d} r|^{2}+\frac{1}{2}\left(\Delta r^{2}\right) \chi_{\nu}\right) \mathrm{e}^{\sigma(r-4 \nu)} \phi\right\|\right\}^{2} \\
\leq & C \nu^{2}\left\|\chi_{\nu / 2}|p \phi|\right\|^{2}+C \nu^{2}\langle\sigma\rangle^{2}\|\phi\|^{2} \\
\leq & C \nu^{2}\left\langle p^{2}\right\rangle_{\phi}+C \nu^{2}\langle\sigma\rangle^{2}\|\phi\|^{2} .
\end{aligned}
$$

Note that $C>0$ does not depend on $\nu$ or $\sigma$ because $r \leq 2 \nu$ on $\operatorname{supp} \chi_{\nu}^{\prime}$. Using the relative $\epsilon$-smallness of the potential we have for some $C>0$

$$
\left\langle p^{2}\right\rangle_{\phi} \leq\langle 4 H+C\rangle_{\phi}=(4 E+C)\|\phi\|^{2}
$$

and we deduce that

$$
-2 \operatorname{Re}\left\langle R(\nu) \mathrm{e}^{\sigma(r-4 \nu)} A \chi_{\nu} \mathrm{e}^{\sigma(r-4 \nu)}\right\rangle_{\phi} \leq C \nu^{2}\langle\sigma\rangle^{2}\|\phi\|^{2} .
$$

On the other hand doing the commutator, cf. (1.13), and then using (1.11a) and (1.14) we obtain that

$$
\begin{aligned}
\left\langle\mathrm{i}\left[H^{\sigma}, A\right]\right\rangle_{\phi_{\sigma}} & \geq-\sigma^{2} \operatorname{Im}\left\langle A|\mathrm{~d} r|^{2}\right\rangle_{\phi_{\sigma}}+2 \sum_{b}\left\langle p_{b}^{2}\right\rangle_{q_{b, R} \phi_{\sigma}}-o\left(R^{0}\right)\left\|\phi_{\sigma}\right\|^{2} \\
& =\sigma^{2}\left\langle r \partial^{r}|\mathrm{~d} r|^{2}\right\rangle_{\phi_{\sigma}}+2 \sum_{b}\left\langle p_{b}^{2}\right\rangle_{q_{b, R} \phi_{\sigma}}-o\left(R^{0}\right)\left\|\phi_{\sigma}\right\|^{2} \\
& \geq \sigma\left\langle\partial^{r}|\mathrm{~d} r|^{2}\right\rangle_{\phi_{\sigma}}+2 \sum_{b}\left\langle p_{b}^{2}\right\rangle_{q_{b, R} \phi_{\sigma}}-o\left(R^{0}\right)\left\|\phi_{\sigma}\right\|^{2}
\end{aligned}
$$

We combine (2.4)-(2.8) and conclude that

$$
\begin{aligned}
& C \nu^{2}\langle\sigma\rangle^{2}\|\phi\|^{2}+o\left(R^{0}\right)\left\|\phi_{\sigma}\right\|^{2} \\
& \geq 4 \sigma\langle r\rangle_{\left(\operatorname{Re} p^{r}\right) \phi_{\sigma}}+2 \sum_{b}\left\langle p_{b}^{2}\right\rangle_{q_{b, R} \phi_{\sigma}} .
\end{aligned}
$$


We aim at deriving some useful positivity from the second term of (2.9) to the right. For that let us for $b \in \mathcal{A}$ introduce

$$
\begin{aligned}
\widetilde{H}_{b} & =\widetilde{H}^{b}+\tilde{p}_{b}^{2} \chi_{\sigma^{2} / 2}^{-}\left(\tilde{p}_{b}^{2}\right) ; \\
s(x) & =\chi_{\nu / 2}^{+}(r)(|\mathrm{d} r|-1) / \sqrt{2}+1 / \sqrt{2}, \\
\tilde{p}_{b}^{2} & =\frac{1}{2} s(x)^{-1} p_{b}^{2} s(x)^{-1}, \\
\widetilde{H}^{b} & =s(x)^{-1} H^{b} s(x)^{-1} .
\end{aligned}
$$

Here we suppressed the dependence of $\widetilde{H}_{b}$ on the parameter $R$ (through $r$, and considered as fixed) as well as the dependence on $\nu$ and $\sigma$. The latter parameters will be considered as independent large parameters (at the end we fix $\nu$ large and let $\sigma \rightarrow \infty)$. The operator $\widetilde{H}_{b}-\sigma^{2}$ should be thought of as an effective approximation to

$$
2|\mathrm{~d} r|^{-1}\left(H_{b}-\frac{\sigma^{2}}{2}|\mathrm{~d} r|^{2}\right)|\mathrm{d} r|^{-1} \approx 2 H_{b}-\sigma^{2}=2 H^{b}+p_{b}^{2}-\sigma^{2} .
$$

Let us here note the following consequence of (1.11c)

$$
\forall \alpha \in \mathbb{N}_{0}^{\operatorname{dim} \mathbf{X}}:\left|\partial_{x}^{\alpha}(s(x)-1 / \sqrt{2})\right| \leq C_{\alpha} \nu^{-2} .
$$

The definitions (2.10) are accompanied by the following specification of domains: For $b \in \mathcal{A}$ we define

$$
\mathcal{H}_{b}=L^{2}\left(\Omega^{b}\right) \otimes L^{2}\left(\mathbf{X}_{b}\right)=L^{2}\left(\Omega^{b}+\mathbf{X}_{b}\right),
$$

and note that

$$
\mathcal{H}_{b} \subset L^{2}\left(\mathbf{X}^{b}\right) \otimes L^{2}\left(\mathbf{X}_{b}\right)=L^{2}(\mathbf{X}) .
$$

The operator $\tilde{p}_{b}^{2}$ is an operator on $L^{2}(\mathbf{X})$ with $Q\left(\tilde{p}_{b}^{2}\right)=Q\left(p_{b}^{2}\right)=L^{2}\left(\mathbf{X}^{b}\right) \otimes H^{1}\left(\mathbf{X}_{b}\right)$ and $\mathcal{D}\left(\tilde{p}_{b}^{2}\right)=\mathcal{D}\left(p_{b}^{2}\right)=L^{2}\left(\mathbf{X}^{b}\right) \otimes H^{2}\left(\mathbf{X}_{b}\right)$. However since it is multiplicative in the $x^{b}$ variable the space $\mathcal{H}_{b}$ is an invariant subspace, in fact $\mathcal{D}\left(\tilde{p}_{b}^{2}\right) \cap \mathcal{H}_{b}=L^{2}\left(\Omega^{b}\right) \otimes H^{2}\left(\mathbf{X}_{b}\right)$. Whence clearly the first term of (2.10) is a bounded operator on $\mathcal{H}_{b}$ (with the norm bound $\frac{7}{8} \sigma^{2}$ ). The second term of $(2.10), \widetilde{H}^{b}$, is also an operator on $\mathcal{H}_{b}$. We specify its form domain to be $L^{2}\left(\mathbf{X}_{b}, H_{0}^{1}\left(\Omega^{b}\right) ; \mathrm{d} x_{b}\right)$. The corresponding quadratic form is closed. We conclude the same for $\widetilde{H}_{b}$, and consequently $\widetilde{H}_{b}$ is a well-defined operator on $\mathcal{H}_{b}$ with

$$
Q\left(\widetilde{H}_{b}\right)=L^{2}\left(\mathbf{X}_{b}, H_{0}^{1}\left(\Omega^{b}\right) ; \mathrm{d} x_{b}\right) \subset \mathcal{H}_{b} .
$$

For later applications let us note the facts that $\mathcal{D}\left(\tilde{p}_{c}^{2}\right) \supset \mathcal{D}\left(\tilde{p}_{b}^{2}\right)$ and $\mathcal{H}_{b} \supset \mathcal{H}_{c}$ for all $c \supset b$ (the latter embedding is due to the relation $\Omega^{b}+\mathbf{X}_{b} \supset \Omega^{c}+\mathbf{X}_{c}$ ).

We introduce a technical condition for the operators introduced in (2.10).

Condition 2.1. For all $b \neq a_{\max }$ the following bound holds uniformly in all large $\sigma, \nu>1, \epsilon \in(0,1]$ and reals $\lambda$ near 1 :

$$
\left\|\delta_{\epsilon}\left(\widetilde{H}_{b} / \sigma^{2}-\lambda\right)\right\|_{\mathcal{B}\left(B\left(\left|x^{b}\right|\right), B\left(\left|x^{b}\right|\right)^{*}\right)} \leq C \sigma .
$$

Here, by definition, for any self-adjoint operator $T$

$$
\delta_{\epsilon}(T)=\pi^{-1} \operatorname{Im}(T-\mathrm{i} \epsilon)^{-1} .
$$

The space $B(\cdot)$ is a Besov space, see Subsection 3.1 for the abstract definition. Note that $(2.12)$ is trivially fulfilled for $b=a_{\text {min }}$ (by the spectral theorem). We derive the bounds for $N=2$ in Section 3 under the additional regularity conditions 
on the obstacles and potentials stated in Theorem 1.6. Note that for $N=2$ and $b \notin\left\{a_{\min }, a_{\max }\right\}$ only $b^{\prime}=b$ obeys $a_{\min } \neq b^{\prime} \subset b$ and hence for such $b$ (2.12) is an effective high energy bound for a bounded obstacle (hence one-body type). More generally we prove (2.12) for $b$ with $\# b=N$ under the additional regularity conditions for $\Omega_{b}$ and $V_{b}$. High energy resolvent bounds are studied previously in the literature, see for example [Je, Vo1, Vo2, RT].

Let us also introduce $\tilde{\phi}_{\sigma}=s(x) \phi_{\sigma}$. We estimate for $b \neq a_{\max }, k_{1}>0$ (can be fixed arbitrarily) and all large $\sigma>1$

$$
\begin{aligned}
\frac{1}{2}\left\langle p_{b}^{2}\right\rangle_{q_{b, R} \phi_{\sigma}} & =\left\langle\tilde{p}_{b}^{2}\right\rangle_{q_{b, R} \tilde{\phi}_{\sigma}} \\
& \geq\left\langle\tilde{p}_{b}^{2} \chi_{k_{1} \sigma}^{+}\left(\tilde{p}_{b}^{2}\right)\right\rangle_{q_{b, R} \tilde{\phi}_{\sigma}} \\
& \geq k_{1} \sigma\left(\left\|q_{b, R} \tilde{\phi}_{\sigma}\right\|^{2}-\left\langle\chi_{k_{1} \sigma}^{-}\left(\tilde{p}_{b}^{2}\right)\right\rangle_{q_{b, R} \tilde{\phi}_{\sigma}}\right) \\
& \geq k_{1} \sigma\left(\left\|q_{b, R} \tilde{\phi}_{\sigma}\right\|^{2}-\left\langle\chi_{\sigma^{2} / 8}^{-}\left(\tilde{p}_{b}^{2}\right)\right\rangle_{q_{b, R} \tilde{\phi}_{\sigma}}\right) .
\end{aligned}
$$

The contribution to (2.9) from the first term to the right in (2.13) amounts (for $\nu \geq R r_{2}$ ) to the positive term $4 k_{1} \sigma\left\|\tilde{\phi}_{\sigma}\right\|^{2}$, and it remains to estimate the contribution from the second term to the right. Now up to a term of order $O\left(\sigma^{-2}\right)$ better it is given by summing the expressions $-4 k_{1} \sigma \operatorname{Re}\left\langle\chi_{\sigma^{2} / 8}^{-}\left(\tilde{p}_{b}^{2}\right) q_{b, R}^{2}\right\rangle_{\tilde{\phi}_{\sigma}}$.

We write for $b \neq a_{\max }$ and $R_{1} \geq R r_{2} / r_{1}$

$$
q_{b, R}^{2}=q_{b, R}^{2} \sum_{b_{1} \supset b} q_{b_{1}, R_{1}}^{2}=q_{b, R}^{2} q_{b, R_{1}}^{2}+q_{b, R}^{2} \sum_{b_{1} \supsetneq b} q_{b_{1}, R_{1}}^{2} .
$$

Actually we shall later need $R_{1}>>R$. We repeat the expansion by writing for $R_{2}>>R_{1}$ and $b_{1} \supsetneq b$

$$
q_{b, R}^{2} q_{b_{1}, R_{1}}^{2}=q_{b, R}^{2} q_{b_{1}, R_{1}}^{2} q_{b_{1}, R_{2}}^{2}+\sum_{b_{2} \supsetneq b_{1}} q_{b, R}^{2} q_{b_{1}, R_{1}}^{2} q_{b_{2}, R_{2}}^{2} .
$$

Upon further iteration the procedure stops for each branch after say $n$ times when necessarily $b_{n}=a_{\max }(n$ is at most $N)$. Whence the only non-trivial terms to examine have the form

$$
q_{b, R}^{2} q_{b, R_{1}}^{2} \text { or } q_{b, R}^{2} \prod_{1 \leq j \leq m} q_{b_{j}, R_{j}}^{2} q_{b_{m}, R_{m+1}}^{2},
$$

where $m \leq n-1, b \subsetneq b_{1} \cdots \subsetneq b_{m} \subsetneq a_{\max }$ and $R<<R_{1} \cdots<<R_{m}<<R_{m+1}$. As the reader will see these constraints are needed later, see the verification of (2.20). Moreover we shall need the constraint $\nu \geq R_{m+1} r_{2}$. Introducing the notation $b_{0}=b$ and $R_{0}=R$ in either case the form is then $q_{b_{m}, R_{m}}^{2} q_{b_{m}, R_{m+1}}^{2}$ times a bounded factor $Q_{m}^{2}$, in fact $\left|Q_{m}(x)\right| \leq 1$. We decompose

$$
\begin{aligned}
& \operatorname{Re}\left(\chi_{\sigma^{2} / 8}^{-}\left(\tilde{p}_{b}^{2}\right) q_{b, R}^{2}\right) \\
& =\operatorname{Re}\left(\sum \chi_{\sigma^{2} / 8}^{-}\left(\tilde{p}_{b}^{2}\right) q_{b_{m}, R_{m}}^{2} q_{b_{m}, R_{m+1}}^{2} Q_{m}^{2}\right)+\operatorname{Re}\left(\sum \chi_{\sigma^{2} / 8}^{-}\left(\tilde{p}_{b}^{2}\right) q_{b_{m}, R_{m}}^{2} q_{a_{\max }, R_{m+1}}^{2} Q_{m}^{2}\right) \\
& =\sum q_{b_{m}, R_{m+1}} q_{b_{m}, R_{m}} Q_{m} \chi_{\sigma^{2} / 8}^{-}\left(\tilde{p}_{b}^{2}\right) Q_{m} q_{b_{m}, R_{m}} q_{b_{m}, R_{m+1}}+\text { remainder. }
\end{aligned}
$$

Here the remainder is the sum of terms either $O\left(\sigma^{-2}\right)$ better than "the good term" $4 k_{1} \sigma\left\|\tilde{\phi}_{\sigma}\right\|^{2}$ we derived from (2.13) or being expressed by factors of $q_{a_{\max }, R_{m+1}}$. Whence (using $\nu \geq R_{m+1} r_{2}$ ) the remainder conforms with (2.9). 
Next on both sides of the factor $\chi_{\sigma^{2} / 8}^{-}\left(\tilde{p}_{b}^{2}\right)$ in the summation to the right we insert

$$
I=\chi_{\sigma^{2} / 4}^{-}\left(\tilde{p}_{b_{m}}^{2}\right)+\chi_{\sigma^{2} / 4}^{+}\left(\tilde{p}_{b_{m}}^{2}\right)
$$

This yields four times as many terms. The contribution from the terms with two factors of $\chi_{\sigma^{2} / 4}^{-}$is then estimated as

$$
\begin{aligned}
& \sum q_{b_{m}, R_{m+1}} q_{b_{m}, R_{m}} Q_{m} \chi_{\sigma^{2} / 4}^{-}\left(\tilde{p}_{b_{m}}^{2}\right) \chi_{\sigma^{2} / 8}^{-}\left(\tilde{p}_{b}^{2}\right) \chi_{\sigma^{2} / 4}^{-}\left(\tilde{p}_{b_{m}}^{2}\right) Q_{m} q_{b_{m}, R_{m}} q_{b_{m}, R_{m+1}} \\
& \leq \operatorname{Re}\left(\sum q_{b_{m}, R_{m+1}}^{2} \chi_{\sigma^{2} / 4}^{-}\left(\tilde{p}_{b_{m}}^{2}\right)^{2} q_{b_{m}, R_{m}}^{2} Q_{m}^{2}\right)+O\left(\sigma^{-2}\right) .
\end{aligned}
$$

We take a closer look at the first term later. We first consider the contributions from

$$
2 \operatorname{Re}\left(\chi_{\sigma^{2} / 4}^{+}\left(\tilde{p}_{b_{m}}^{2}\right) \chi_{\sigma^{2} / 8}^{-}\left(\tilde{p}_{b}^{2}\right) \chi_{\sigma^{2} / 4}^{-}\left(\tilde{p}_{b_{m}}^{2}\right)\right)+\chi_{\sigma^{2} / 4}^{+}\left(\tilde{p}_{b_{m}}^{2}\right) \chi_{\sigma^{2} / 8}^{-}\left(\tilde{p}_{b}^{2}\right) \chi_{\sigma^{2} / 4}^{+}\left(\tilde{p}_{b_{m}}^{2}\right) .
$$

For this purpose let us for $\psi \in L^{2}(\mathbf{X})$ introduce

$$
\psi_{\sigma}=\chi_{\sigma^{2} / 4}^{+}\left(\tilde{p}_{b_{m}}^{2}\right) \chi_{\sigma^{2} / 8}^{-}\left(\tilde{p}_{b}^{2}\right) \psi \text { and } \widetilde{\psi}_{\sigma}=\chi_{\sigma^{2} / 8}^{-}\left(\tilde{p}_{b}^{2}\right) \chi_{\sigma^{2} / 4}^{+}\left(\tilde{p}_{b_{m}}^{2}\right) \psi
$$

We apply (see below for a proof)

$$
\left\|\left[\chi_{\sigma^{2} / 4}^{+}\left(\tilde{p}_{b_{m}}^{2}\right), \chi_{\sigma^{2} / 8}^{-}\left(\tilde{p}_{b}^{2}\right)\right]\right\| \leq C \frac{1}{\sigma \nu}
$$

yielding

$$
\left\|\psi_{\sigma}-\widetilde{\psi}_{\sigma}\right\| \leq C \frac{1}{\sigma \nu}\|\psi\|
$$

and whence with the operator monotone function $f(t)=(t-1) /(1+t)$

$$
\begin{aligned}
\left\|\widetilde{\psi}_{\sigma}\right\|^{2} & \leq 2\left\|\psi_{\sigma}\right\|^{2}+C \frac{1}{\sigma^{2} \nu^{2}}\|\psi\|^{2} \\
& \leq 2 f\left(\frac{5}{4}\right)^{-1}\left\langle f\left(\frac{4}{\sigma^{2}} \tilde{p}_{b_{m}}^{2}\right)\right\rangle_{\psi_{\sigma}}+C \frac{1}{\sigma^{2} \nu^{2}}\|\psi\|^{2} \\
& \leq 2 f\left(\frac{5}{4}\right)^{-1}\left\langle f\left(\frac{4}{\sigma^{2}} \tilde{p}_{b}^{2}\right)\right\rangle_{\psi_{\sigma}}+C \frac{1}{\sigma^{2} \nu^{2}}\|\psi\|^{2} .
\end{aligned}
$$

Obviously it follows from (2.15a) that

$$
\left\|f\left(\frac{4}{\sigma^{2}} \tilde{p}_{b}^{2}\right)\left[\chi_{\sigma^{2} / 4}^{+}\left(\tilde{p}_{b_{m}}^{2}\right), \chi_{\sigma^{2} / 8}^{-}\left(\tilde{p}_{b}^{2}\right)\right]\right\| \leq C \frac{1}{\sigma \nu},
$$

and we can "reverse the commutation"

$$
\begin{aligned}
2 f\left(\frac{5}{4}\right)^{-1} & \left\langle f\left(\frac{4}{\sigma^{2}} \tilde{p}_{b}^{2}\right)\right\rangle_{\psi_{\sigma}} \\
& \leq 2 f\left(\frac{5}{4}\right)^{-1}\left\langle f\left(\frac{4}{\sigma^{2}} \tilde{p}_{b}^{2}\right)\right\rangle_{\widetilde{\psi}_{\sigma}}+C \frac{1}{\sigma \nu}\|\psi\|\left\|\psi_{\sigma}\right\|+C \frac{1}{\sigma \nu}\|\psi\|\left\|\widetilde{\psi}_{\sigma}\right\| \\
& \leq 2 f\left(\frac{5}{4}\right)^{-1} f\left(\frac{7}{8}\right)\left\|\widetilde{\psi}_{\sigma}\right\|^{2}+\left\|\psi_{\sigma}\right\|^{2}+\epsilon\left\|\widetilde{\psi}_{\sigma}\right\|^{2}+C_{\epsilon} \frac{1}{\sigma^{2} \nu^{2}}\|\psi\|^{2} \\
& \leq\left\|\psi_{\sigma}\right\|^{2}+C_{\epsilon} \frac{1}{\sigma^{2} \nu^{2}}\|\psi\|^{2}
\end{aligned}
$$

here we took $\epsilon=2 f\left(\frac{5}{4}\right)^{-1}\left|f\left(\frac{7}{8}\right)\right|$, for example. By combining with the previous estimation we find

$$
\left\|\psi_{\sigma}\right\|^{2} \leq C \frac{1}{\sigma^{2} \nu^{2}}\|\psi\|^{2}
$$

and then in turn

$$
\left\|\psi_{\sigma}\right\|,\left\|\widetilde{\psi_{\sigma}}\right\| \leq C \frac{1}{\sigma \nu}\|\psi\| .
$$

We conclude that indeed, due to errors of the form $O\left(\nu^{-1} \sigma^{-1}\right)+O\left(\sigma^{-2}\right)$, we need to examine the first term of (2.14) only. 
2.1.1. Verification of (2.15a). Introduce $P_{m}=\sigma^{-2} \tilde{p}_{b_{m}}^{2}$ and $P=\sigma^{-2} \tilde{p}_{b}^{2}$. We show the slightly stronger bound

$$
\left\|\left[\chi_{1 / 4}^{+}\left(P_{m}\right), \chi_{1 / 8}^{-}(P)\right]\right\|=\left\|\left[\chi_{1 / 4}^{-}\left(P_{m}\right), \chi_{1 / 8}^{-}(P)\right]\right\| \leq C \frac{1}{\sigma \nu^{2}} .
$$

Since $P_{m}, P \geq 0$ we can truncate $\chi_{\nu}^{-}, \nu=1 / 4,1 / 8$, at the negative half-axis to become functions $\chi_{1}, \chi_{2}$ in $C_{\mathrm{c}}^{\infty}(\mathbb{R})$ and invoke the standard representation for a self-adjoint operator $T$ and such function $\chi$

$$
\chi(T)=\int_{\mathbb{C}}(T-z)^{-1} \mathrm{~d} \mu(z), \mathrm{d} \mu(z)=-\frac{1}{2 \pi \mathrm{i}} \bar{\partial} \tilde{\chi}(z) \mathrm{d} z \mathrm{~d} \bar{z},
$$

where we have used an almost analytic extension $\tilde{\chi} \in C_{\mathrm{c}}^{\infty}(\mathbb{C})$, i.e.

$$
\tilde{\chi}(t)=\chi(t) \text { for } t \in \mathbb{R}, \quad|\bar{\partial} \tilde{\chi}(z)| \leq C_{k}|\operatorname{Im} z|^{k} ; k \in \mathbb{N} .
$$

Whence

$$
\begin{aligned}
\chi_{1 / 4}^{-}\left(P_{m}\right) & =\int_{\mathbb{C}}\left(P_{m}-z_{1}\right)^{-1} \mathrm{~d} \mu_{1}\left(z_{1}\right), \\
\chi_{1 / 8}^{-}(P) & =\int_{\mathbb{C}}\left(P-z_{2}\right)^{-1} \mathrm{~d} \mu_{2}\left(z_{2}\right) .
\end{aligned}
$$

Using (2.17a), (2.17b) and the domain relation $\mathcal{D}\left(P_{m}\right) \supset \mathcal{D}(P)$ we represent

$$
\begin{aligned}
{\left[\chi_{1 / 4}^{-}\left(P_{m}\right), \chi_{1 / 8}^{-}(P)\right]=\int_{\mathbb{C}} \int_{\mathbb{C}}\left(P_{m}-z_{1}\right)^{-1}\left(P-z_{2}\right)^{-1} } & {\left[P_{m}, P\right]\left(P-z_{2}\right)^{-1}\left(P_{m}-z_{1}\right)^{-1} \mathrm{~d} \mu_{2}\left(z_{2}\right) \mathrm{d} \mu_{1}\left(z_{1}\right) . }
\end{aligned}
$$

Next we note the elementary bounds

$$
\begin{aligned}
\left\|\left(P_{m}-z\right)^{-1}\right\| & \leq \frac{1}{|\operatorname{Im} z|}, \\
\left\|\langle P\rangle(P-z)^{-1}\right\| & \leq C \frac{|z|+1}{|\operatorname{Im} z|} .
\end{aligned}
$$

Using (2.11) we compute

$$
\left\|\langle P\rangle^{-1}\left[P_{m}, P\right]\langle P\rangle^{-1}\right\| \leq C \frac{1}{\sigma \nu^{2}} .
$$

Finally applying (2.18a)-(2.18c) to the double integral we obtain the bound

$$
\cdots \leq C_{1} \frac{1}{\sigma \nu^{2}} \int_{\mathbb{C}} \int_{\mathbb{C}}\left|\operatorname{Im} z_{1}\right|^{-2} \frac{\left(\left|z_{2}\right|+1\right)^{2}}{\left|\operatorname{Im} z_{2}\right|^{2}}\left|\mathrm{~d} \mu_{2}\left(z_{2}\right)\right|\left|\mathrm{d} \mu_{1}\left(z_{1}\right)\right|=C_{2} \frac{1}{\sigma \nu^{2}},
$$

and we have shown $(2.15 \mathrm{c})$.

2.1.2. Localization for first term of (2.14). We decompose (for $k_{2}>0$ to be fixed later, big)

$$
\begin{aligned}
q_{b_{m}, R_{m+1}}^{2} \chi_{\sigma^{2} / 4}^{-}\left(\tilde{p}_{b_{m}}^{2}\right)^{2} q_{b_{m}, R_{m}}^{2} Q_{m}^{2} & =q_{b_{m}, R_{m+1}}^{2} \chi_{\sigma^{2} / 4}^{-}\left(\tilde{p}_{b_{m}}^{2}\right)\left(\chi^{-}+\chi^{+}\right) q_{b_{m}, R_{m}}^{2} Q_{m}^{2} \\
\chi^{-} & :=\chi_{\left(k_{2} R_{m} \sigma\right)^{-1}}^{-}\left(\left|\widetilde{H}_{b_{m}} / \sigma^{2}-1\right|\right), \\
\chi^{+} & :=\chi_{\left(k_{2} R_{m} \sigma\right)^{-1}}^{+}\left(\left|\widetilde{H}_{b_{m}} / \sigma^{2}-1\right|\right) .
\end{aligned}
$$

To treat the contribution from $\chi^{-}$we write $q_{b_{m}, R_{m}}^{2}=\chi_{r_{2} R_{m}}^{-}\left(\left|x^{b_{m}}\right|\right) q_{b_{m}, R_{m}}^{2}$. Now imposing Condition 2.1 and applying (2.12) with $b=b_{m}$ we get using

$$
\left\|\chi_{r_{2} R_{m}}^{-}\left(\left|x^{b_{m}}\right|\right)\right\|_{\mathcal{B}\left(\mathcal{H}_{b_{m}}, B\left(\left|x^{b_{m}}\right|\right)\right)} \leq C \sqrt{r_{2} R_{m}}
$$

that

$$
\left\|\chi_{r_{2} R_{m}}^{-}\left(\left|x^{b_{m}}\right|\right)\left(\chi^{-}\right)^{2} \chi_{r_{2} R_{m}}^{-}\left(\left|x^{b_{m}}\right|\right)\right\|_{\mathcal{B}\left(\mathcal{H}_{b_{m}}\right)} \leq C_{1} / k_{2}
$$


Here we used the general bound for $S$ bounded and $T$ self-adjoint

$$
\left\|S^{*} g(T) S\right\| \leq\|g\|_{L^{1}} \sup _{\lambda \in \operatorname{supp} g, \epsilon \in(0,1]}\left\|S^{*} \delta_{\epsilon}(T-\lambda) S\right\|,
$$

cf. Stone's formula [RS]. We fix $k_{2}$ such that $(\# \mathcal{A})^{N+1} \sqrt{C_{1} / k_{2}} \leq 1 / 2$, saving "the good term" $2 k_{1} \sigma\left\|\tilde{\phi}_{\sigma}\right\|^{2}$ in the previous bound $(2.9)$.

2.1.3. Completion of proof of (2.1). We need to examine the contribution from $\chi^{+}$ to $(2.9)$. We write

$$
q_{b_{m}, R_{m+1}}^{2} \chi_{\sigma^{2} / 4}^{-}\left(\tilde{p}_{b_{m}}^{2}\right)^{2} \chi^{+}=k_{2} R_{m} \sigma q_{b_{m}, R_{m+1}}^{2} \chi_{\sigma^{2} / 4}^{-}\left(\tilde{p}_{b_{m}}^{2}\right)^{2}\left(\widetilde{H}_{b_{m}} / \sigma^{2}-1\right) \widetilde{Q}_{m},
$$

where $\widetilde{Q}_{m}=\widetilde{Q}_{m}\left(\widetilde{H}_{b_{m}} / \sigma^{2}\right)$ is bounded with norm at most 1 . Taking expectation in $\tilde{\phi}_{\sigma}$ and using the Cauchy Schwarz inequality it suffices to bound

$$
\begin{aligned}
& 4 k_{1} \sigma \sum k_{2} R_{m} \sigma^{-1}\left\|\left(\widetilde{H}_{b_{m}}-\sigma^{2}\right) \chi_{\sigma^{2} / 4}^{-}\left(\tilde{p}_{b_{m}}^{2}\right)^{2} q_{b_{m}, R_{m+1}}^{2} \tilde{\phi}_{\sigma}\right\|\left\|\tilde{\phi}_{\sigma}\right\| \\
& \leq k_{1} \sigma\left\|\tilde{\phi}_{\sigma}\right\|^{2}+4 \sigma\langle r\rangle_{\left(\operatorname{Re} p^{r}\right) \phi_{\sigma}}+C\left(\nu^{2}\langle\sigma\rangle^{2}\|\phi\|^{2}+\left\|\phi_{\sigma}\right\|^{2}\right) .
\end{aligned}
$$

With (2.9) this yields

$$
C\left(\nu^{2}\langle\sigma\rangle^{2}\|\phi\|^{2}+\left\|\phi_{\sigma}\right\|^{2}\right) \geq k_{1} \sigma\left(1-O\left(\nu^{-1} \sigma^{-1}\right)-O\left(\sigma^{-2}\right)\right)\left\|\tilde{\phi}_{\sigma}\right\|^{2},
$$

and we learn by letting $\sigma \rightarrow \infty$ that $\chi_{4 \nu}(r) \phi \equiv 0$ (for $\nu$ large), and then in turn from the unique continuation property that $\phi=0$.

2.1.4. Mapping properties. As a preparation for proving (2.20) let us note the following mapping properties of $S=\chi_{\sigma^{2} / 4}^{-}\left(\tilde{p}_{a}^{2}\right)^{2}$ and $T=q_{a, R_{m+1}}^{2}$ entering in $(2.20)$ with $a=b_{m}$. Recall that the form domain $Q\left(\widetilde{H}_{a}\right)$ of $\widetilde{H}_{a}$ is $L^{2}\left(\mathbf{X}_{a}, H_{0}^{1}\left(\Omega^{a}\right) ; \mathrm{d} x_{a}\right)$.

$$
\begin{array}{r}
S \in \mathcal{B}\left(Q\left(\widetilde{H}_{a}\right), H_{0}^{1}\left(\Omega^{a}+\mathbf{X}_{a}\right)\right), \\
T \in \mathcal{B}\left(H_{0}^{1}\left(\Omega^{a}+\mathbf{X}_{a}\right), H_{0}^{1}(\Omega)\right), \\
T S \in \mathcal{B}\left(Q\left(\widetilde{H}_{a}\right), H_{0}^{1}(\Omega)\right), \\
T S \in \mathcal{B}\left(Q\left(\widetilde{H}_{a}\right)\right) .
\end{array}
$$

For (2.21a) we can use (2.16) to represent $S=\chi\left(\tilde{p}_{a}^{2}\right)$, apply the integral to a simple tensor $\psi^{a} \otimes \psi_{a}$ and then calculate derivatives of the resulting expression (not to be elaborated on). Clearly $S$ is a smoothing operator in the $x_{a}$ variable yielding the improved smoothness. Also we note that since $S$ is multiplicative in the $x^{a}$ variable it preserves the support in this variable of elements of an approximating sequence. We obtain that indeed $S \psi_{a} \otimes \psi^{a} \in H_{0}^{1}\left(\Omega^{a}+\mathbf{X}_{a}\right)$ with

$$
\left\|S \psi^{a} \otimes \psi_{a}\right\|_{1} \leq C\left\|\psi^{a} \otimes \psi_{a}\right\|_{Q\left(\widetilde{H}_{a}\right)} .
$$

This bound extends to finite sums of simple tensors (by the same arguments) and hence $(2.21 \mathrm{a})$ follows by density and continuity. As for $(2.21 \mathrm{~b})$ we use that

$$
\operatorname{supp}\left(q_{a, R_{m+1}}^{2}\right) \cap\left(\Omega^{a}+\mathbf{X}_{a}\right) \subset \Omega .
$$

Clearly (2.21c) follows from (2.21a) and (2.21b), while in turn (2.21d) follows from (2.21c) and the inclusion $\Omega \subset \Omega^{a}+\mathbf{X}_{a}$ implying that $H_{0}^{1}(\Omega)$ is continuously embedded in $H_{0}^{1}\left(\Omega^{a}+\mathbf{X}_{a}\right)$ and therefore in $Q\left(\widetilde{H}_{a}\right)$. 
2.1.5. Proof of (2.20). We consider the vector $\left(\widetilde{H}_{a}-\sigma^{2}\right) \chi_{\sigma^{2} / 4}^{-}\left(\tilde{p}_{a}^{2}\right)^{2} q_{a, R_{m+1}}^{2} \tilde{\phi}_{\sigma}, a=b_{m}$, in $(2.20)$ as an element of the dual space of the form domain $Q\left(\widetilde{H}_{a}\right)$, that is in $L^{2}\left(\mathbf{X}_{a}, H_{0}^{1}\left(\Omega^{a}\right)^{*} ; \mathrm{d} x_{a}\right)$. As a part of $(2.20)$ we must show that indeed it belongs to $\mathcal{H}_{a}$. This will follow from $(2.21 \mathrm{c})$ and the calculations below. We rewrite, using that $\tilde{\phi}_{\sigma} \in Q\left(\widetilde{H}_{a}\right), \widetilde{H}_{a} \tilde{\phi}_{\sigma} \in Q\left(\widetilde{H}_{a}\right)^{*}$ and $(2.21 \mathrm{~d})$,

$$
\begin{aligned}
\left(\widetilde{H}_{a}-\sigma^{2}\right) \chi_{\sigma^{2} / 4}^{-}\left(\tilde{p}_{a}^{2}\right)^{2} q_{a, R_{m+1}}^{2} \tilde{\phi}_{\sigma} & =\chi_{\sigma^{2} / 4}^{-}\left(\tilde{p}_{a}^{2}\right)^{2} q_{a, R_{m+1}}^{2}\left(\widetilde{H}_{a}-\sigma^{2}\right) \tilde{\phi}_{\sigma}+T_{\mathrm{com}} \\
T_{\mathrm{com}} & =\left[\widetilde{H}_{a}, \chi_{\sigma^{2} / 4}^{-}\left(\tilde{p}_{a}^{2}\right)^{2} q_{a, R_{m+1}}^{2}\right] \tilde{\phi}_{\sigma},
\end{aligned}
$$

and then

$$
\begin{aligned}
\left(\widetilde{H}_{a}-\sigma^{2}\right) \tilde{\phi}_{\sigma} & =s(x)^{-1}\left(\frac{1}{2} p_{a}^{2} s(x)^{-1} \chi_{\sigma^{2} / 2}^{-}\left(\tilde{p}_{a}^{2}\right) s(x)+H^{a}-\frac{\sigma^{2}}{2}|\mathrm{~d} r|^{2}\right) \phi_{\sigma} \\
& =s(x)^{-1}\left(\left(H^{\sigma}-E+\mathrm{i} \sigma\left(\operatorname{Re} p^{r}\right)\right) \phi_{\sigma}+\mathrm{ie}^{\sigma(r-4 \nu)} R(\nu) \phi\right)+T_{1}+T_{2}+T_{3} \\
T_{1} & =-\tilde{p}_{a}^{2} \chi_{\sigma^{2} / 2}^{+}\left(\tilde{p}_{a}^{2}\right) \tilde{\phi}_{\sigma}, \\
T_{2} & =s(x)^{-1}\left(E-I_{a}-\mathrm{i} \sigma\left(\operatorname{Re} p^{r}\right)\right) \phi_{\sigma} \\
T_{3} & =-s(x)^{-1} \mathrm{ie}^{\sigma(r-4 \nu)} R(\nu) \phi .
\end{aligned}
$$

Due to $(2.3)$ (and $(2.21 \mathrm{c})$ ) we need to estimate the contributions from $T_{1}-T_{3}$ and $T_{\text {com }}$ only.

As for $T_{1}$ we note that $\left(\chi_{\sigma^{2} / 4}^{-}\right)^{2} \chi_{\sigma^{2} / 2}^{+}=0$. Whence by commutation

$$
\left\|\chi_{\sigma^{2} / 4}^{-}\left(\tilde{p}_{a}^{2}\right)^{2} q_{a, R_{m+1}}^{2} T_{1}\right\| \leq C \frac{\sigma}{R_{m+1}}\left\|\tilde{\phi}_{\sigma}\right\|
$$

which agrees with $(2.20)$ provided $R_{m+1}>>R_{m}$.

We estimate

$$
\begin{aligned}
\left\|\chi_{\sigma^{2} / 4}^{-}\left(\tilde{p}_{a}^{2}\right)^{2} q_{a, R_{m+1}}^{2} T_{2}\right\|^{2} & \leq C\left\|\phi_{\sigma}\right\|^{2}+2 \sigma^{2}\left\|\left(\operatorname{Re} p^{r}\right) \phi_{\sigma}\right\|^{2} \\
& \leq\left(C+O\left(\sigma^{2} / \nu^{2}\right)\right)\left\|\phi_{\sigma}\right\|^{2}+\frac{2}{\nu} \sigma^{2}\langle r\rangle_{\left(\operatorname{Re} p^{r}\right) \phi_{\sigma}} .
\end{aligned}
$$

Whence the contribution from $T_{2}$ to the bound (2.20) is given by

$$
\cdots \leq C \epsilon^{-1} R_{m}\left(\left(\sigma^{-1}+\frac{\sigma}{\nu^{2}}\right)\left\|\phi_{\sigma}\right\|^{2}+\frac{\sigma}{\nu}\langle r\rangle_{\left(\operatorname{Re} p^{r}\right) \phi_{\sigma}}\right)+\epsilon R_{m} \sigma\left\|\tilde{\phi}_{\sigma}\right\|^{2} .
$$

This bound agrees with (2.20) for all large $\nu$ and $\sigma$ if we choose $\epsilon>0$ small (note that $\nu>>R_{m}$ is used). Notice that we needed the second term on the right hand side of (2.20) (this is the only occurrence).

As for the contribution from $T_{3}$ we invoke (2.6).

To treat the contribution from $T_{\text {com }}$ we decompose

$$
T_{\text {com }}=\chi_{\sigma^{2} / 4}^{-}\left(\tilde{p}_{a}^{2}\right)^{2}\left[\tilde{H}_{a}, q_{a, R_{m+1}}^{2}\right] \tilde{\phi}_{\sigma}+\left[\widetilde{H}_{a}, \chi_{\sigma^{2} / 4}^{-}\left(\tilde{p}_{a}^{2}\right)^{2}\right] q_{a, R_{m+1}}^{2} \tilde{\phi}_{\sigma}
$$

and use the representation (2.16) for both terms to the right.

Noting the following generalization of (2.6)

$$
\left\|\chi_{\nu} \mathrm{e}^{\sigma r}|p \phi|\right\|^{2} \leq 4 E\left\|\chi_{\nu} \mathrm{e}^{\sigma r} \phi\right\|^{2}+C\langle\sigma\rangle^{2}\left\|\chi_{\nu / 2} \mathrm{e}^{\sigma r} \phi\right\|^{2},
$$

it follows (for the first term) that

$$
\left\|\left[\widetilde{H}_{a}, q_{a, R_{m+1}}^{2}\right] \tilde{\phi}_{\sigma}\right\| \leq C \frac{\sigma}{R_{m+1}}\left(\left\|\tilde{\phi}_{\sigma}\right\|+\|\phi\|\right),
$$

which agrees with $(2.20)$ provided $R_{m+1}>>R_{m}$. 
We claim that

$$
\left\|\left[\widetilde{H}_{a}, \chi_{\sigma^{2} / 4}^{-}\left(\tilde{p}_{a}^{2}\right)^{2}\right] q_{a, R_{m+1}}^{2} \tilde{\phi}_{\sigma}\right\| \leq C \frac{\sigma}{\nu}\left(\left\|\tilde{\phi}_{\sigma}\right\|+\|\phi\|\right),
$$

which also agrees with (2.20), hence finally showing the latter bound.

Now for showing (2.23) we do various commutation using (2.22)

$$
\begin{aligned}
& {\left[\widetilde{H}_{a}, \chi_{\sigma^{2} / 4}^{-}\left(\tilde{p}_{a}^{2}\right)^{2}\right] q_{a, R_{m+1}}^{2} \tilde{\phi}_{\sigma}} \\
& =O\left(\frac{\sigma}{\nu}\right)+\left[s(x)^{-2}, \chi_{\sigma^{2} / 4}^{-}\left(\tilde{p}_{a}^{2}\right)^{2}\right] H^{a} q_{a, R_{m+1}}^{2} \tilde{\phi}_{\sigma} \\
& =O\left(\frac{\sigma}{\nu}\right)+O\left(\frac{1}{\sigma \nu}\right)\left\langle\sigma^{-2} \tilde{p}_{a}^{2}\right\rangle^{-1} s(x)^{-1} H^{a} q_{a, R_{m+1}}^{2} \phi_{\sigma} \\
& =O\left(\frac{\sigma}{\nu}\right)+O\left(\frac{1}{\nu R_{m+1}}\right)+O\left(\frac{1}{\sigma \nu}\right)\left\langle\sigma^{-2} \tilde{p}_{a}^{2}\right\rangle^{-1} s(x)^{-1} q_{a, R_{m+1}}^{2} H^{a} \phi_{\sigma},
\end{aligned}
$$

where we used the convention $\left\|O\left(\frac{\sigma}{\nu}\right)\right\| \leq C \frac{\sigma}{\nu}\left(\left\|\tilde{\phi}_{\sigma}\right\|+\|\phi\|\right)$ and similarly for the term $O\left(\frac{1}{\nu R_{m+1}}\right)$. Next we decompose

$$
\begin{aligned}
H^{a} \phi_{\sigma} & =\left(\left(H^{\sigma}-E+\mathrm{i} \sigma\left(\operatorname{Re} p^{r}\right)\right) \phi_{\sigma}+\mathrm{ie}^{\sigma(r-4 \nu)} R(\nu) \phi\right) \\
& +\left(E-\mathrm{i} \sigma\left(\operatorname{Re} p^{r}\right)+\frac{\sigma^{2}}{2}|\mathrm{~d} r|^{2}-\frac{1}{2} p_{a}^{2}-I_{a}\right) \phi_{\sigma}-\mathrm{ie}^{\sigma(r-4 \nu)} R(\nu) \phi .
\end{aligned}
$$

The first term vanishes. The second term contributes with a term of the form $O\left(\frac{\sigma}{\nu}\right)$. To see this we use (2.22) in two applications (note that the factor $\left\langle\sigma^{-2} \tilde{p}_{a}^{2}\right\rangle^{-1} s(x)^{-1}$ is used to bound one factor of $p_{a}^{2}$ ), and we use the factor $q_{a, R_{m+1}}^{2}$ (note that $q_{a, R_{m+1}}^{2} I_{a}$ is bounded). The third term is $O\left(\frac{1}{\nu}\right)$. We have shown $(2.23)$.

\section{High-ENERGY HARD-CORE ONE-BODY RESOLVENT BOUND}

In Subsections 3.3-3.5 we verify Condition 2.1 for $N=2$ under Conditions 1.4 and 1.5. The proof will be based on various results for abstract Besov spaces to be given in Subsection 3.1 and on a variant of Mourre theory somewhat related to [Sk3]. We present our main results for the obstacle case in Subsection 3.2. These will be given in a slightly more general setting, and we devote Subsections 3.3 and 3.4 to proofs. The case of an empty obstacle is treated in Subsection 3.5.

3.1. Abstract Besov spaces. Let $A$ be a self-adjoint operator on a Hilbert space $\mathcal{H}$. Let $R_{0}=0$ and $R_{j}=2^{j-1}$ for $j \in \mathbb{N}$. We define correspondingly characteristic functions $F_{j}=F\left(R_{j-1} \leq|\cdot|<R_{j}\right)$ and the space

$$
B=B(A)=\left\{u \in \mathcal{H} \mid \sum_{j \in \mathbb{N}} R_{j}^{1 / 2}\left\|F_{j}(A) u\right\|=:\|u\|_{B}<\infty\right\} .
$$

We can identify (using the embeddings $\langle A\rangle^{-1} \mathcal{H} \subset B \subset \mathcal{H} \subset B^{*},\langle A\rangle:=\sqrt{A^{2}+1}$ ) the dual space $B^{*}$ as

$$
B^{*}=B(A)^{*}=\left\{u \in\langle A\rangle \mathcal{H} \mid \sup _{j \geq 1} R_{j}^{-1 / 2}\left\|F_{j}(A) u\right\|=:\|u\|_{B^{*}}<\infty\right\} .
$$

Alternatively, the elements $u$ of $B^{*}$ are those sequences $u=\left(u_{j}\right) \subset \mathcal{H}$ with $u_{j} \in$ $\operatorname{Ran}\left(F_{j}(A)\right)$ and $\sup _{j \in \mathbb{N}} R_{j}^{-1 / 2}\left\|u_{j}\right\|<\infty$. For previous related works we refer to [AH, JP, GY, Wa, Ro, Sk3] and [Hö, Subsections 14.1 and 30.2]. We note the bounds, cf. [Hö, Subsections 14.1],

$$
\|u\|_{B^{*}} \leq \sup _{R>1} R^{-1 / 2}\|F(|A|<R) u\| \leq 2\|u\|_{B^{*}}
$$


Introducing abstract weighted spaces $L_{s}^{2}=L_{s}^{2}(A)=\langle A\rangle^{-s} \mathcal{H}$ we have the embeddings

$$
L_{s}^{2} \subset B \subset L_{1 / 2}^{2} \subset \mathcal{H} \subset L_{-1 / 2}^{2} \subset B^{*} \subset L_{-s}^{2} \text {, for all } s>1 / 2 .
$$

All embeddings are continuous and corresponding bounding constants can be chosen as absolute constants, i.e. independently of $A$ and $\mathcal{H}$. In particular

$$
\|u\|_{\mathcal{H}} \leq\|u\|_{B} \text { for all } u \in B \text {. }
$$

We refer to the spaces $B$ and $B^{*}$ as abstract Besov spaces. Recall the following interpolation type result, here stated abstractly. The proof is the same as that of the concrete versions [AH, Theorem 2.5], [Hö, Theorem 14.1.4], [JP, Proposition 2.3] and [Ro, Subsection 4.3].

Lemma 3.1. Let $A_{1}$ and $A_{2}$ be self-adjoint operators on Hilbert spaces $\mathcal{H}_{1}$ and $\mathcal{H}_{2}$, respectively, and let $s>1 / 2$. Suppose $T \in \mathcal{B}\left(\mathcal{H}_{1}, \mathcal{H}_{2}\right) \cap \mathcal{B}\left(L_{s}^{2}\left(A_{1}\right), L_{s}^{2}\left(A_{2}\right)\right)$. Then $T \in \mathcal{B}\left(B\left(A_{1}\right), B\left(A_{2}\right)\right)$, and there is a constant $C=C(s)>0$ (independent of $T$ ) such that

$$
\|T\|_{\mathcal{B}\left(B\left(A_{1}\right), B\left(A_{2}\right)\right)} \leq C\left(\|T\|_{\mathcal{B}\left(\mathcal{H}_{1}, \mathcal{H}_{2}\right)}+\|T\|_{\mathcal{B}\left(L_{s}^{2}\left(A_{1}\right), L_{s}^{2}\left(A_{2}\right)\right)}\right) .
$$

We state and prove the following (partial) version of [Sk3, Lemma 2.5].

Lemma 3.2. Suppose $A$ is a self-adjoint operator on a Hilbert space $\mathcal{H}, c>1$ and $u \in B(A)$, then $u \in B(c A)$ with

$$
\|u\|_{B(c A)} \leq 8 c^{1 / 2}\|u\|_{B(A)} .
$$

Proof. Pick $i \geq 2$ such that $R_{i-1}<c \leq R_{i}$. Then for all $j \geq i+1$

$$
F_{j}(c t) \leq F\left(R_{j-1} / R_{i} \leq|t|<R_{j} / R_{i-1}\right) \leq F_{j-i+1}(t)+F_{j-i+2}(t) .
$$

Whence for any $u \in B(A)$ we can estimate

$$
\begin{aligned}
\|u\|_{B(c A)} & \leq\left(\sup _{j \geq i+1}\left(R_{j} / R_{j-i+1}\right)^{1 / 2}+\sup _{j \geq i+1}\left(R_{j} / R_{j-i+2}\right)^{1 / 2}\right)\|u\|_{B(A)}+\sum_{j=1}^{i} R_{j}^{1 / 2}\|u\|_{\mathcal{H}} \\
& \leq\left(2^{(i-1) / 2}+2^{(i-2) / 2}+2^{i / 2}(\sqrt{2}+1)\right)\|u\|_{B(A)} \\
& \leq(\sqrt{2}+1+2(\sqrt{2}+1)) c^{1 / 2}\|u\|_{B(A)} \\
& \leq 8 c^{1 / 2}\|u\|_{B(A)} .
\end{aligned}
$$

We note the following abstract version of a result from [JP, Mo2] (proven by using suitable decompositions of unity and the Cauchy Schwarz inequality, see also [Wa, Subsection 2.2]).

Lemma 3.3. Let $A_{1}$ and $A_{2}$ be self-adjoint operators on Hilbert spaces $\mathcal{H}_{1}$ and $\mathcal{H}_{2}$, respectively, and let $T \in \mathcal{B}\left(\mathcal{H}_{1}, \mathcal{H}_{2}\right)$. Suppose that uniformly in $m, n \in \mathbb{Z}$,

$$
\left\|F\left(m \leq A_{2}<m+1\right) T F\left(n \leq A_{1}<n+1\right)\right\| \leq C .
$$

Then with the constant $C$ from (3.8) we have

$$
\|T\|_{\mathcal{B}\left(B\left(A_{1}\right), B\left(A_{2}\right)^{*}\right)} \leq 2 C .
$$

We note the following (partial) abstract criterion for (3.8), cf. [Mo2, (I.10)] (see also [Wa]). Recall that a bounded operator $T$ on a Hilbert space is called accretive if $T+T^{*} \geq 0$, cf. for example [RS, Chapter X]. 
Lemma 3.4. Let $A$ be a self-adjoint operator on a Hilbert space $\mathcal{H}$, and suppose $T \in \mathcal{B}(\mathcal{H})$ is accretive. Suppose the following bounds uniformly in $n \in \mathbb{Z}$,

$$
\begin{aligned}
\| F(n \leq & A<n+1) T F(n \leq A<n+1) \| \leq C_{1}, \\
\|F(A<n) T F(n \leq A<n+1)\| & \leq C_{2}, \\
& \|F(n \leq A<n+1) T F(A \geq n)\| \leq C_{3} .
\end{aligned}
$$

Then (3.8) holds with $A_{1}=A_{2}=A$, the accretive $T$ and with $C=2 C_{1}+C_{2}+C_{3}$.

3.2. Setting of problem. We assume that $\Omega \subset \mathbf{X}$ is open and that $\Theta:=\mathbf{X} \backslash \bar{\Omega} \neq \emptyset$ is bounded with smooth boundary $\partial \Theta=\partial \Omega$. Moreover we assume that $\Theta$ is strictly convex, see Appendix B for definition. The case $\Omega=\mathbf{X}$ is simpler and will be treated in Subsection 3.5.

We consider a Hilbert space $\mathcal{H}=L^{2}(\Omega, \mathrm{d} x) \otimes L^{2}(M, \mathrm{~d} y)$. The structure of the second factor will not be of importance. To make contact to (2.12) we think of $x$ as $x^{b}$ and $y$ as $x_{b}$ (here $b \notin\left\{a_{\min }, a_{\max }\right\}$ and $\left.\# b=N\right)$. Hence the function $s$ of $(2.12)$ is now a function of $x$ and $y$, viz. $s=s(x, y)$. The operator $\widetilde{H}_{b}$ takes the form $\widetilde{H}_{b}=\tilde{H}^{b}+\tilde{B}_{b}$ on $\mathcal{H}$. We simplify notation and look at

$$
\begin{aligned}
H & =\widetilde{H}^{b}+\widetilde{B} \\
\widetilde{H}^{b} & =s(x, y)^{-1}\left(\frac{1}{2} p_{x}^{2}+V(x)\right) s(x, y)^{-1}=\tilde{p}_{x}^{2}+V(x) s(x, y)^{-2}, \\
\widetilde{B} & =\widetilde{B}(x),
\end{aligned}
$$

where the operator $\widetilde{B}(x)$ acts as a bounded operator on the component $L^{2}(M, \mathrm{~d} y)$. As an operator on $\mathcal{H}$ it is bounded, and it needs to be small and regular in $x$ in a certain sense (to be specified in (3.19c)-(3.19e)). Whence our method does not require much specific structure of the operator-valued potential $\widetilde{B}$. The unbounded part, $\widetilde{H}^{b}$, is defined with Dirichlet boundary condition at $\partial \Omega$, and the two-body potential $V=V(x)$ needs to be sufficiently regular. For simplicity we impose $V \in C^{\infty}(\bar{\Omega})$ and $\partial_{x}^{\alpha} V(x)=O\left(|x|^{-\varepsilon-|\alpha|}\right)$, cf. Condition 1.4 (1). Whence the form domain of $\widetilde{H}^{b}$ is given by the space

$$
Q\left(\widetilde{H}^{b}\right)=Q(H)=L^{2}\left(M, H_{0}^{1}(\Omega) ; \mathrm{d} y\right) \subset \mathcal{H} .
$$

The reader should keep in mind the rough approximation $\widetilde{H}^{b} \approx-\Delta_{x}+2 V(x)$ (recall here that $s \approx 1 / \sqrt{2}$ in the large $\nu$ regime). We denote the resolvent of $H_{\sigma}:=\sigma^{-2} H$ by $R(z, \sigma)$, viz. $R(z, \sigma)=\left(H_{\sigma}-z\right)^{-1}$.

We introduce a function $r=r(x)$ that is different from the function $r$ of Section 2. It is now given as

$$
r(x)=\operatorname{dist}(x, \partial \Omega),
$$

which can be extended to a smooth function on $\mathbf{X}$ and which at infinity has bounds

$$
\partial^{\alpha} r=O\left(r^{1-|\alpha|}\right)=O\left(\langle x\rangle^{1-|\alpha|}\right) .
$$

More importantly there exists $c>0$ such that

$$
\nabla^{2} r_{\mid\{r(x)=r\}} \geq \frac{c}{1+r} I .
$$


The verification of (3.11b) and (3.11c) is given in Appendix B. Note the following consequence of (3.11c),

$$
\forall \delta \in(0,1]: \frac{\nabla^{2} r^{2}}{2} \geq \delta \mathrm{d} r \otimes \mathrm{d} r \oplus r \nabla^{2} r_{\mid\{r(x)=r\}} \geq \min (\delta, c) \frac{r}{1+r} I .
$$

In terms of the function $r$ we introduce a conjugate operator different from the operator $A$ that appears in Lemma 1.3. Now

$$
A:=\frac{\nabla r^{2}}{2} \cdot p+p \cdot \frac{\nabla r^{2}}{2} .
$$

This operator is self-adjoint on $L^{2}(\Omega, \mathrm{d} x)$ (it is essentially self-adjoint on $C_{\mathrm{c}}^{\infty}(\Omega)$ ) and whence also on $\mathcal{H}$. Note that $Q(H)$ is "boundedly stable" under the dynamics generated by $A$ (using here terminology of [GGM], see also [FMS]), i.e.

$$
\forall \psi \in Q(H): \sup _{|t|<1}\left\|\mathrm{e}^{\mathrm{i} t A} \psi\right\|_{Q(H)}<\infty .
$$

We note the representation $A=r p_{r}+p_{r} r$ where

$$
p_{r}:=\frac{\nabla r}{2} \cdot p+p \cdot \frac{\nabla r}{2}=-\mathrm{i} \frac{\partial}{\partial r}-\mathrm{i} \frac{\Delta r}{2} .
$$

In turn the operator $p_{r}$ is symmetric as an operator with domain $H_{0}^{1}(\Omega)$, and we define $p_{r}^{2}$ as the Friedrichs extension from $C_{\mathrm{c}}^{2}(\Omega)$ and use the same notation for $p_{r}^{2} \otimes I$. Note the inclusion $Q(H) \subset Q\left(p_{r}^{2}\right)$ for form domains as well as the following analogue of (3.13a)

$$
\forall \psi \in Q\left(p_{r}^{2}\right): \sup _{|t|<1}\left\|\mathrm{e}^{\mathrm{i} t A} \psi\right\|_{Q\left(p_{r}^{2}\right)}<\infty .
$$

Note for (3.13a) and (3.13b) that a similar property is derived in Appendix A for the conjugate operator used in Section 2 (the one constructed by the Graf vector field). Note for (3.13b) the explicit formula $\left\|p_{r} \mathrm{e}^{\mathrm{i} t A} \psi\right\|=\mathrm{e}^{2 t}\left\|p_{r} \psi\right\|$. For a different proof, given in a generalized setting, see Lemma A.11. The property (3.13a) follows from Lemma A.7.

We recall the Hardy bounds, cf. [Da, Lemma 5.3.1],

$$
\left\|r^{-\kappa}\left|p_{r}\right|^{-\kappa}\right\| \leq 2 \text { for } \kappa \in[0,1] \text {. }
$$

Moreover we have

$$
-\Delta_{x}=p_{r}^{2}+L^{2}+\frac{1}{4}(\Delta r)^{2}+\frac{1}{2}\left(\partial_{r} \Delta r\right)
$$

where the second term is positive and commutes with $r$ (it is the Laplace-Beltrami operator in geodesic coordinates), and the third and fourth terms are bounded functions on $\Omega$.

We also introduce operators $f_{1}, f_{2} \geq 0$ with squares

$$
\begin{aligned}
& f_{1}^{2}=\sigma^{-2 / 3}+\frac{r}{1+r}, \\
& f_{2}^{2}=\sigma^{-2 / 3}+\frac{r}{1+r}+\sigma^{-2} p_{r}^{2}=f_{1}^{2}+\sigma^{-2} p_{r}^{2} .
\end{aligned}
$$

A main preliminary bound of this section is

Lemma 3.5. With A given by (3.12) we have uniformly in all large $\sigma, \nu>1$ and all $\operatorname{Re} z \approx 1$

$$
\left\|f_{2} R(z, \sigma) f_{2}\right\|_{\mathcal{B}\left(B(A), B(A)^{*}\right)} \leq C .
$$

The main result of the section is 
Proposition 3.6. With $r$ given as the multiplication operator on $\mathcal{H}$ in terms of the function (3.11a) we have uniformly in all large $\sigma, \nu>1$ and all $\operatorname{Re} z \approx 1$

$$
\|R(z, \sigma)\|_{\mathcal{B}\left(B(r), B(r)^{*}\right)} \leq C \sigma .
$$

Obviously for $b$ with $\# b=N$ and $\Omega_{b} \neq \mathbf{X}^{b}$, and under the regularity conditions on $\Omega=\Omega_{b}$ and $V_{b}=V$ introduced above, the bound (2.12) is a consequence of Proposition 3.6.

3.3. Besov space bound of resolvent, Lemma 3.5. In this subsection we shall prove Lemma 3.5 using a variant of Mourre theory.

3.3.1. First order commutator. We "compute" the commutator

$$
\begin{aligned}
\mathrm{i}[H, A]:= & s^{-1}\left(2 p_{r}^{2}+2 p_{i} r\left(\nabla^{2} r\right)^{i j} p_{j}+W\right) s^{-1} \\
& +2 \operatorname{Re}\left(s^{-1}\left(\nabla r^{2} \cdot \nabla_{x} s\right) \widetilde{H}^{b}\right)-\nabla r^{2} \cdot \nabla_{x} \widetilde{B} ; \\
W(x):= & \frac{1}{2}(\Delta r)^{2}+\partial_{r} \Delta r-\frac{1}{4} \Delta^{2} r^{2}-\nabla r^{2} \cdot \nabla V(x) .
\end{aligned}
$$

Thus at this stage the first order commutator $\mathrm{i}[H, A]$ is defined by its formal expression. We note that it is a bounded quadratic form on $Q(H)$. The term $W$ is a bounded function, and the second and third terms are "small". More precisely in terms of the parameters $\nu$ and $\sigma$ of Section 2 we have uniform bounds, cf. (1.11c), (2.11) and (2.16),

$$
\begin{aligned}
\left|\frac{1+r}{r s} \nabla r^{2} \cdot \nabla_{x} s\right| & \leq C \nu^{-1}, \\
\left|\frac{1+r}{r}\left(\nabla r^{2} \cdot \nabla_{x}\right)^{2} s\right| & \leq C, \\
\left\|\frac{1+r}{r} \nabla r^{2} \cdot \nabla_{x} \widetilde{B}\right\| & \leq C \frac{\sigma^{2}}{\nu}, \\
\left\|\frac{1+r}{r}\left(\nabla r^{2} \cdot \nabla_{x}\right)^{2} \widetilde{B}\right\| & \leq C \sigma^{2}, \\
0 \leq \widetilde{B} & \leq \frac{7}{8} \sigma^{2} .
\end{aligned}
$$

We can estimate the second term after commutation as

$$
2 \operatorname{Re}\left(s^{-1}\left(\nabla r^{2} \cdot \nabla_{x} s\right) \widetilde{H}^{b}\right) \geq-C_{1} \nu^{-1}\left(\operatorname{Re}\left(\frac{r}{1+r} H\right)+C_{2}\right),
$$

cf. (3.19a) and (3.19e).

Similarly we can estimate the third term as

$$
-\nabla r^{2} \cdot \nabla_{x} \widetilde{B} \geq-C \frac{\sigma^{2}}{\nu} \frac{r}{1+r}
$$

cf. $(3.19 \mathrm{c})$.

Using these bounds, (3.11c) and (3.14) we can estimate for some small $\delta>0$ (and uniformly in $\sigma, \nu>1$ )

$$
\begin{aligned}
\mathrm{i}\left[\sigma^{-2} H, A\right] & \geq \sigma^{-2} 3 p_{r}^{2}+\delta\left(\sigma^{-2} r^{-2}+\operatorname{Re}\left(\frac{r}{1+r} \sigma^{-2} \widetilde{H}^{b}\right)\right) \\
& -C_{1} \nu^{-1} \operatorname{Re}\left(\frac{r}{1+r} \sigma^{-2} H\right)-C_{3}\left(\sigma^{-2}+\nu^{-1} \frac{r}{1+r}\right) .
\end{aligned}
$$

Next we estimate using (3.19e)

$$
\operatorname{Re}\left(\frac{r}{1+r} \sigma^{-2} \widetilde{H}^{b}\right) \geq\left(\operatorname{Re} z-\frac{7}{8}\right) \frac{r}{1+r}+\operatorname{Re}\left(\frac{r}{1+r}\left(\sigma^{-2} H-z\right)\right) .
$$


We are interested in the regime $\operatorname{Re} z \approx 1$. Concretely let us assume that $\mid 1-$ $\operatorname{Re} z \mid \leq \frac{1}{9}$ allowing us to estimate uniformly in the spectral parameter: There exists $\delta^{\prime} \in(0,3)$ such that for all such $z$ and all large $\sigma, \nu>1$

$$
\delta\left(\sigma^{-2} r^{-2}+\left(\operatorname{Re} z-\frac{7}{8}\right) \frac{r}{1+r}\right)-C_{1} \nu^{-1} \operatorname{Re} z \frac{r}{1+r}-C_{3}\left(\sigma^{-2}+\nu^{-1} \frac{r}{1+r}\right) \geq \delta^{\prime} f_{1}^{2} .
$$

From (3.20) we thus obtain

$$
\mathrm{i}\left[H_{\sigma}, A\right] \geq \delta^{\prime} f_{2}^{2}+\left(\delta-C_{1} \nu^{-1}\right) \operatorname{Re}\left(\frac{r}{1+r}\left(H_{\sigma}-z\right)\right) .
$$

Now let us introduce (cf. the method of [Mo1])

$$
R_{z}(\epsilon)=\left(H_{\sigma}-\mathrm{i} \epsilon \mathrm{i}\left[H_{\sigma}, A\right]-z\right)^{-1} ; \epsilon \operatorname{Im} z>0,|1-\operatorname{Re} z| \leq \frac{1}{9} .
$$

We only need $|\epsilon| \leq 1$, and we note that as a form $H_{\sigma}-\mathrm{i} \in \mathrm{i}\left[H_{\sigma}, A\right]$ is strictly $m$ sectorial in the terminology of [Ka, RS], cf. the computation (3.18). The associated operator, cf. [RS, Theorem VIII.17], is invertible if we also assume that $|\operatorname{Im} z|>>1$, and hence the inverse is well-defined with adjoint $R_{z}(\epsilon)^{*}=R_{\bar{z}}(-\epsilon)$ under these conditions. However it follows from a connectedness argument and $(3.23 \mathrm{~b})$ stated below (with $T=f_{2}$ ) that $R_{z}(\epsilon)$ is well-defined without the condition $|\operatorname{Im} z|>>1$. Note also that $\lim _{\epsilon \rightarrow 0} R_{z}(\epsilon)=R(z, \sigma)$.

We obtain from (3.21a) that

$$
\mathrm{i}\left[H_{\sigma}, A\right] \geq \frac{\delta^{\prime}}{2} f_{2}^{2}+\left(\delta-C_{1} \nu^{-1}\right) \operatorname{Re}\left(\frac{r}{1+r}\left(H_{\sigma}-\mathrm{i} \in \mathrm{i}\left[H_{\sigma}, A\right]-z\right)\right) .
$$

In fact using (3.15) and (3.18) we compute

$$
\begin{aligned}
& \operatorname{Re}\left(\frac{r}{1+r} \mathrm{i} \epsilon \mathrm{i}\left[H_{\sigma}, A\right]\right) \\
& =\frac{\epsilon}{2 \sigma^{2}} \mathrm{i}\left[\frac{r}{1+r}, \mathrm{i}[H, A]\right] \\
& =\frac{\epsilon}{2 \sigma^{2}} \mathrm{i}\left[\frac{r}{1+r}, s^{-1} 2 p_{r}^{2} s^{-1}+\operatorname{Re}\left(s^{-1}\left(\nabla r^{2} \cdot \nabla_{x} s\right) s^{-1} p_{r}^{2} s^{-1}\right)\right] \\
& =-\frac{\epsilon}{\sigma^{2}} s^{-1}\left(2 \operatorname{Re}\left(p_{r}(1+r)^{-2}\right)+\operatorname{Re}\left(\left(\nabla r^{2} \cdot \nabla_{x} s\right) s^{-1} \operatorname{Re}\left(p_{r}(1+r)^{-2}\right)\right)\right) s^{-1} \\
& =-\frac{\epsilon}{\sigma^{2}} \operatorname{Re}\left(p_{r} g\right)
\end{aligned}
$$

where $g=g(x, y)$ is a uniformly bounded function, and thus indeed

$$
-\left(\delta-C_{1} \nu^{-1}\right) \operatorname{Re}\left(\frac{r}{1+r} \mathrm{i} \epsilon \mathrm{i}\left[H_{\sigma}, A\right]\right) \leq C \sigma^{-2 / 3} f_{2}^{2} \leq \frac{\delta^{\prime}}{2} f_{2}^{2} .
$$

Due to (3.21b) and the second resolvent equation we have the quadratic estimate

$$
\left\|f_{2} R_{z}(\epsilon) T\right\|^{2} \leq C_{1}\left(|\epsilon|^{-1}\left\|T^{*} R_{z}(\epsilon) T\right\|+\left\|T^{*} R_{z}(\epsilon)^{*} \frac{r}{1+r} T\right\|\right) .
$$

Hence if $T$ is an operator obeying

$$
\left\|f_{2}^{-1} \frac{r}{1+r} T\right\| \leq C_{2}
$$

then

$$
\left\|f_{2} R_{z}(\epsilon) T\right\|^{2} \leq C_{1}\left(|\epsilon|^{-1}\left\|T^{*} R_{z}(\epsilon) T\right\|+C_{2}\left\|T^{*} R_{z}(\epsilon)^{*} f_{2}\right\|\right) .
$$

This leads to

$$
\left\|f_{2} R_{z}(\epsilon) T\right\|^{2} \leq C_{3}|\epsilon|^{-1}\left\|T^{*} R_{z}(\epsilon) T\right\|+C_{4} .
$$


We have the examples $T=f_{2}$ and $T=f_{2}\langle A\rangle^{-1}$ with bounds independent of all large $\sigma$ and $\nu$. Indeed for all $\psi \in \mathcal{H}$ (or alternatively for all $\psi \in L^{2}(\Omega, \mathrm{d} x)$ since the operators act on the first tensor factor only)

$$
\begin{aligned}
\left\langle f_{2}^{2}\right\rangle_{\frac{r}{1+r} f_{2}^{-1} \psi} & \leq\|\psi\|^{2}+\left\langle\sigma^{-2} p_{r}^{2}\right\rangle_{\frac{r}{1+r}} f_{2}^{-1} \psi \\
& \leq\|\psi\|^{2}+\left\|\sigma^{-1} p_{r} f_{2}^{-1} \psi\right\|^{2}+C_{1} \sigma^{-2}\left\|f_{2}^{-1} \psi\right\|^{2} \\
& \leq C_{2}\|\psi\|^{2}
\end{aligned}
$$

proving (3.23a) for these examples.

3.3.2. Technical lemma. For $B \in \mathcal{B}\left(Q\left(p_{r}^{2}\right), \mathcal{H}\right)$ we define

$$
\operatorname{ad}_{A}(B)=[B, A]=\mathrm{s}_{t \rightarrow 0}^{-} \operatorname{limit}^{-1}\left(B \mathrm{e}^{-\mathrm{i} t A}-\mathrm{e}^{-\mathrm{i} t A} B\right)_{\mid Q\left(p_{r}^{2}\right) \rightarrow \mathcal{H}},
$$

provided the right hand side exists. Note that we here use (3.13b). In the terminology of $[\mathrm{GGM}], B \in C^{1}\left(A_{\mid Q\left(p_{r}^{2}\right)}, A_{\mid \mathcal{H}}\right)$ if the right hand side exists. We use this interpretation of the (repeated) commutators in the following lemma (in turn to be used later).

Lemma 3.7. Uniformly in all $\sigma>1$

$$
\begin{aligned}
& \left\|\operatorname{ad}_{A}\left(f_{2}\right) f_{2}^{-1}\right\| \leq C, \\
& \left\|\operatorname{ad}_{A}^{2}\left(f_{2}\right) f_{2}^{-1}\right\| \leq C .
\end{aligned}
$$

Proof. Note the representation (valid for any strictly positive operator $S$ )

$$
S^{-1 / 2}=\pi^{-1} \int_{0}^{\infty} s^{-1 / 2}(S+s)^{-1} \mathrm{~d} s .
$$

With $S=f_{2}^{2}$ we thus obtain (for the first term)

$$
\begin{aligned}
{\left[f_{2}, A\right] } & =S^{-1 / 2}[S, A]+\left[S^{-1 / 2}, A\right] S \\
& =S^{-1 / 2}[S, A]-\pi^{-1} \int_{0}^{\infty} s^{-1 / 2}(S+s)^{-1}[S, A](S+s)^{-1} \mathrm{~d} s S
\end{aligned}
$$

where

$$
[S, A]=\mathrm{s}_{t \rightarrow 0}^{-\lim _{t \rightarrow 0}} \mathrm{it}^{-1}\left(S \mathrm{e}^{-\mathrm{i} t A}-\mathrm{e}^{-\mathrm{i} t A} S\right)_{\mid Q\left(p_{r}^{2}\right) \rightarrow Q\left(p_{r}^{2}\right)^{*}}
$$

is computed (up a factor $-\mathrm{i}$ ) as

$$
\mathrm{i}[S, A]=2 \operatorname{Re}\left(\mathrm{i}\left[S, r p_{r}\right]\right)=4 \sigma^{-2} p_{r}^{2}-2 r(1+r)^{-2}=4 S-4 f_{1}^{2}-2 r(1+r)^{-2} .
$$

In particular

$$
-C S \leq \mathrm{i}[S, A] \leq C S,
$$

which obviously allows us to conclude that

$$
B_{1}:=S^{-1 / 2}[S, A] S^{-1 / 2}
$$

is bounded. Similarly we introduce

$$
B_{2}:=\int_{0}^{\infty} s^{-1 / 2}(S+s)^{-1}[S, A](S+s)^{-1} \mathrm{~d} s S^{1 / 2},
$$


and it remains to show boundedness of $B_{2}$ : We write with $f:=\left(f_{1}^{2}+\frac{1}{2} r(1+r)^{-2}\right)^{1 / 2}$

$$
\begin{aligned}
B_{2} & =C I+\mathrm{i} \int_{0}^{\infty} s^{-1 / 2}(S+s)^{-1} 4 f^{2}(S+s)^{-1} \mathrm{~d} s S^{1 / 2} \\
& =C\left(I-f^{2} S^{-1}\right)+\mathrm{i} \int_{0}^{\infty} s^{-1 / 2}\left[(S+s)^{-1}, 4 f^{2}\right](S+s)^{-1} \mathrm{~d} s S^{1 / 2} \\
& =C\left(I-f S^{-1} f-f\left[f, S^{-1}\right]\right)-4 \mathrm{i} \int_{0}^{\infty} s^{-1 / 2}(S+s)^{-1}\left[S, f^{2}\right](S+s)^{-2} \mathrm{~d} s S^{1 / 2} \\
& =B-C f S^{-1}[S, f] S^{-1}-4 \mathrm{i} \int_{0}^{\infty} s^{-1 / 2}(S+s)^{-1}\left[S, f^{2}\right](S+s)^{-2} \mathrm{~d} s S^{1 / 2} .
\end{aligned}
$$

Using the notation $O(1)=O_{\mathcal{B}(\mathcal{H})}\left(\sigma^{0}\right)$ we have

$$
\begin{aligned}
\mathrm{i}[S, f] & =\frac{p_{r}}{\sigma} O(1)(\sigma f)^{-1}+\text { h.c., } \\
\mathrm{i}\left[S, f^{2}\right] & =\frac{p_{r}}{\sigma} O(1) \sigma^{-1}+\text { h.c. }=\sigma^{-2 / 3} S^{1 / 2} O(1) S^{1 / 2},
\end{aligned}
$$

and the first identity yields that also

$$
\text { if } \begin{aligned}
S^{-1}[S, f] S^{-1} & =\left(f S^{-1} \frac{p_{r}}{\sigma}\right) O(1)(\sigma f)^{-1} S^{-1}+f S^{-1}\left(\sigma^{2 / 3} f\right)^{-1} O(1)\left(\sigma^{-1 / 3} \frac{p_{r}}{\sigma} S^{-1}\right) \\
& =O(1) \sigma^{-2 / 3} S^{-1}+f S^{-1} \sigma^{-1 / 3} O(1)=O(1) ;
\end{aligned}
$$

i.e. the term is uniformly bounded. The second identity yields that the integral is bounded by

$$
\begin{aligned}
& C \sigma^{-2 / 3} \int_{0}^{\infty} s^{-1 / 2}\left\|(S+s)^{-1} S^{1 / 2}(S+s)^{-1}\right\| \mathrm{d} s \\
& \leq C \sigma^{-2 / 3} \int_{0}^{\infty} s^{-1 / 2}\left(\sigma^{-2 / 3}+s\right)^{-3 / 2} \mathrm{~d} s
\end{aligned}
$$

and since the latter integral is independent of $\sigma$ indeed also the integral is uniformly bounded. So also $B_{2}$ is uniformly bounded and (3.24a) follows.

As for (3.24b) we use a previous computation to obtain

$$
\begin{aligned}
-\operatorname{ad}_{A}^{2}(S) & =4\left(4 S-4 f_{1}^{2}-2 r(1+r)^{-2}\right)+2 r \frac{\partial}{\partial r}\left(4 f_{1}^{2}+2 r(1+r)^{-2}\right) \\
& =16 S-16 \tilde{f}^{2} ; \tilde{f}=\left(\sigma^{-2 / 3}+\frac{\frac{3}{4} r+\frac{9}{4} r^{2}+r^{3}}{(1+r)^{3}}\right)^{1 / 2} .
\end{aligned}
$$

which leads to form-boundedness

$$
\left\|S^{-1 / 2} \operatorname{ad}_{A}^{2}(S) S^{-1 / 2}\right\| \leq C .
$$

We decompose

$$
\begin{aligned}
\operatorname{ad}_{A}^{2}\left(f_{2}\right) S^{-1 / 2} & =T_{1}+\cdots+T_{5} ; \\
T_{1} & =\operatorname{ad}_{A}\left(S^{-1 / 2}\right) \operatorname{ad}_{A}(S) S^{-1 / 2}=\left(\operatorname{ad}_{A}\left(S^{-1 / 2}\right) S^{1 / 2}\right)\left(S^{-1 / 2} \operatorname{ad}_{A}(S) S^{-1 / 2}\right), \\
T_{2} & =S^{-1 / 2} \operatorname{ad}_{A}^{2}(S) S^{-1 / 2} \\
T_{3} & =-\pi^{-1} \int_{0}^{\infty} s^{-1 / 2}(S+s)^{-1} \operatorname{ad}_{A}^{2}(S)(S+s)^{-1} \mathrm{~d} s S^{1 / 2}, \\
T_{4} & =\pi^{-1} \int_{0}^{\infty} s^{-1 / 2}(S+s)^{-1} \operatorname{ad}_{A}(S)(S+s)^{-1} \operatorname{ad}_{A}(S)(S+s)^{-1} \mathrm{~d} s S^{1 / 2}, \\
T_{5} & =-\pi^{-1} \int_{0}^{\infty} s^{-1 / 2}(S+s)^{-1} \operatorname{ad}_{A}(S)(S+s)^{-1} \operatorname{ad}_{A}(S)(S+s)^{-1} s \mathrm{~d} s S^{-1 / 2} .
\end{aligned}
$$


The boundedness of the term $T_{1}$ follows from the previous proof. Clearly the term $T_{2}$ is bounded. We can show boundedness of $T_{3}$ as we proceeded for (3.24a) (note that now $\tilde{f}$ plays the role of the previous $f$ ). For $T_{4}$ and $T_{5}$ we rewrite

$$
\begin{aligned}
T_{4}+T_{5} & =\widetilde{T}_{4}+\widetilde{T}_{5} \\
\widetilde{T}_{4} & =\pi^{-1} \int_{0}^{\infty} s^{-1 / 2}(S+s)^{-1} \operatorname{ad}_{A}(S)(S+s)^{-1} \mathrm{~d} s S^{1 / 2}\left(S^{-1 / 2} \operatorname{ad}_{A}(S) S^{-1 / 2}\right), \\
\widetilde{T}_{5} & =-2 \pi^{-1} \int_{0}^{\infty} s^{-1 / 2}(S+s)^{-1} \operatorname{ad}_{A}(S)(S+s)^{-1} \operatorname{ad}_{A}(S)(S+s)^{-1} s \mathrm{~d} s S^{-1 / 2} .
\end{aligned}
$$

The boundedness of the term $\widetilde{T}_{4}$ follows from the previous proof. Whence it only remains to show boundedness of $\widetilde{T}_{5}$. We proceed in a similar fashion as before substituting for the first factor of $\operatorname{ad}_{A}(S)$ from the left $\operatorname{ad}_{A}(S)=-\mathrm{i} 4\left(S-f^{2}\right)$ and then move to the left. The commutator is treated as in (3.25) using now also the form-boundedness of the second factor of $\operatorname{ad}_{A}(S)$. So it remains to consider

$$
\left(I-f^{2} S^{-1}\right) \int_{0}^{\infty} s^{-1 / 2} S(S+s)^{-2} \operatorname{ad}_{A}(S)(S+s)^{-1} S^{-1 / 2} s \mathrm{~d} s .
$$

We saw before that the first factor is bounded. For the integral we substitute again $\operatorname{ad}_{A}(S)=-\mathrm{i} 4\left(S-f^{2}\right)$ and move to the left. Estimating as in (3.25) we conclude that the commutator is bounded. So we are left with

$$
\int_{0}^{\infty} \cdots \mathrm{d} s=C_{1}\left(S-f^{2}\right) \int_{0}^{\infty} s^{-1 / 2} S(S+s)^{-3} S^{-1 / 2} s \mathrm{~d} s=C_{2}\left(I-f^{2} S^{-1}\right),
$$

which is bounded. Whence (3.24b) follows.

3.3.3. Second order commutator. In (3.18) we took the formal commutator as a definition of $\mathrm{i}[H, A]$, however due to the property (3.13a) there is the following alternative interpretation

$$
-\mathrm{i}[H, A]=\mathrm{s}_{t \rightarrow 0}^{-\lim _{t \rightarrow}} t^{-1}\left(H \mathrm{e}^{-\mathrm{i} t A}-\mathrm{e}^{-\mathrm{i} t A} H\right)_{\mid Q(H) \rightarrow Q(H)^{*}},
$$

cf. Appendix A and [GGM], which allows us to compute

$$
\frac{\mathrm{d}}{\mathrm{d} \epsilon} R_{z}(\epsilon)=-R_{z}(\epsilon)\left[H_{\sigma}, A\right] R_{z}(\epsilon)=R_{z}(\epsilon) A-A R_{z}(\epsilon)+\epsilon R_{z}(\epsilon) \operatorname{ad}_{A}^{2}\left(H_{\sigma}\right) R_{z}(\epsilon),
$$

where $\operatorname{ad}_{A}^{2}\left(H_{\sigma}\right)=\left[\left[H_{\sigma}, A\right], A\right] \in \mathcal{B}\left(Q(H), Q(H)^{*}\right)$. Note that in the terminology of [GGM], $H \in C^{2}\left(A_{\mid Q(H)}, A_{\mid Q(H)^{*}}\right)$. The second identity of (3.26) is valid as a form on the domain $\mathcal{D}^{*}:=\mathcal{D}\left(A_{\mid Q(H)^{*}}\right)$ of the generator of the extended group $\left\{\mathrm{e}^{-\mathrm{i} t A}\right\}_{\mid Q(H)^{*}}$, so that indeed $A: \mathcal{D}^{*} \rightarrow Q(H)^{*}$, which combines with the mapping property $R_{z}(\epsilon): Q(H)^{*} \rightarrow Q(H)$. Below we use tacitly this interpretation and the fact that $f_{2}\langle A\rangle^{-1}: \mathcal{H} \rightarrow \mathcal{D}^{*}$, cf. (3.24a).

Using (3.14) (with $\kappa=1 / 2),(3.18)$ and (3.19a)-(3.19d) we compute

$$
\operatorname{ad}_{A}^{2}\left(H_{\sigma}\right)=f_{2} B_{0} f_{2}+\sum_{i, j=1}^{\operatorname{dim} \mathbf{X}}\left(\frac{r}{1+r}\right)^{1 / 2}(\sigma s)^{-1} p_{i} B_{i j} p_{j}(\sigma s)^{-1}\left(\frac{r}{1+r}\right)^{1 / 2}
$$

where $p_{j}$ denotes the components of $p_{x}$ and all $B$ 's are uniformly bounded. 
Using (3.23b), (3.26) and (3.27) we shall prove three bounds which are uniform in $z$ and $\epsilon$ as specified above and (for convenience) with $\operatorname{Im} z, \epsilon>0$ as well as uniform in (large) $\sigma$ and $\nu$ :

$$
\begin{aligned}
\left\|F_{z}(\epsilon)\right\| & \leq C \text { for } F_{z}(\epsilon):=\langle A\rangle^{-1} f_{2} R_{z}(\epsilon) f_{2}\langle A\rangle^{-1} \\
\left\|F_{z}^{-}(\epsilon)\right\| & \leq C \text { for } F_{z}^{-}(\epsilon):=\mathrm{e}^{\epsilon A} F(A<0) f_{2} R_{z}(\epsilon) f_{2}\langle A\rangle^{-2} \\
\left\|F_{z}^{+}(\epsilon)\right\| & \leq C \text { for } F_{z}^{+}(\epsilon):=\langle A\rangle^{-2} f_{2} R_{z}(\epsilon) f_{2} F(A \geq 0) \mathrm{e}^{-\epsilon A} .
\end{aligned}
$$

$\operatorname{Re}(3.28 \mathrm{a})$. Due to (3.23b) for $T=f_{2}\langle A\rangle^{-1}$ (note that we proved (3.23a))

$$
\left\|F_{z}(\epsilon)\right\| \leq C \epsilon^{-1} \text { for } 0<\epsilon \leq 1 .
$$

Obviously (3.24a) yields the bounds

$$
\left\|f_{2}^{-1} A f_{2}\langle A\rangle^{-1}\right\| \leq C \text { and }\left\|\langle A\rangle^{-1} f_{2} A f_{2}^{-1}\right\| \leq C
$$

Exploiting (3.23b), (3.26), (3.27) and (3.30) we can show that

$$
\left\|\frac{\mathrm{d}}{\mathrm{d} \epsilon} F_{z}(\epsilon)\right\| \leq C\left(\epsilon^{-1 / 2}\left\|F_{z}(\epsilon)\right\|^{1 / 2}+\left\|F_{z}(\epsilon)\right\|+\widetilde{C}\right) .
$$

Here we can argue as follows for the contribution to (3.26) from the second term in (3.27). For $\psi \in \mathcal{H}$ we estimate using (3.14), (3.19e) and (3.22)

$$
\begin{gathered}
\sum_{j}\left\|p_{j}(\sigma s)^{-1}\left(\frac{r}{1+r}\right)^{1 / 2} \psi\right\|^{2} \leq C_{1}\left\|f_{2} \psi\right\|^{2}+2 \operatorname{Re}\left\langle H_{\sigma}-z\right\rangle_{r^{1 / 2}(1+r)^{-1 / 2} \psi} \\
\leq C_{2}\left\|f_{2} \psi\right\|^{2}+2 \operatorname{Re}\left\langle\frac{r}{1+r}\left(H_{\sigma}-\mathrm{i} \in \mathrm{i}\left[H_{\sigma}, A\right]-z\right\rangle_{\psi} .\right.
\end{gathered}
$$

We use (3.32a) to $\psi=R_{z}(\epsilon) f_{2}\langle A\rangle^{-1} \tilde{\psi}, \tilde{\psi} \in \mathcal{H}$, and then (3.23a) and (3.23b) with $T=f_{2}\langle A\rangle^{-1}$. Similarly we apply

$$
\sum_{i}\left\|p_{i}(\sigma s)^{-1}\left(\frac{r}{1+r}\right)^{1 / 2} \psi\right\|^{2} \leq C_{2}\left\|f_{2} \psi\right\|^{2}+2 \operatorname{Re}\left\langle\frac{r}{1+r}\left(H_{\sigma}+\mathrm{i} \epsilon \mathrm{i}\left[H_{\sigma}, A\right]-\bar{z}\right\rangle_{\psi}\right.
$$

to $\psi=R_{\bar{z}}(-\epsilon) f_{2}\langle A\rangle^{-1} \tilde{\psi}$. We conclude $(3.31)$.

Clearly (3.28a) follows from (3.29) and (3.31) by two integrations.

Re (3.28b). Due to (3.23b) and (3.28a)

$$
\left\|F_{z}^{-}(\epsilon)\right\| \leq C \epsilon^{-1 / 2} \text {. }
$$

Using (3.26) we compute

$$
\begin{aligned}
\frac{\mathrm{d}}{\mathrm{d} \epsilon} F_{z}^{-}(\epsilon) & =T_{1}+T_{2}+T_{3} \\
T_{1} & =\mathrm{e}^{\epsilon A} F(A<0)\left[A, f_{2}\right] R_{z}(\epsilon) f_{2}\langle A\rangle^{-2}, \\
T_{2} & =\mathrm{e}^{\epsilon A} F(A<0) f_{2} R_{z}(\epsilon) A f_{2}\langle A\rangle^{-2}, \\
T_{3} & =\epsilon \mathrm{e}^{\epsilon A} F(A<0) f_{2} R_{z}(\epsilon) \operatorname{ad}_{A}^{2}\left(H_{\sigma}\right) R_{z}(\epsilon) f_{2}\langle A\rangle^{-2} .
\end{aligned}
$$

Using again (3.23b) and (3.28a) we can estimate

$$
\left\|T_{j}\right\| \leq C \epsilon^{-1 / 2} \text { for } 0<\epsilon \leq 1 \text { and } j=1,2,3 .
$$

Notice that for all of the terms $T_{1}-T_{3}$ we apply (3.23b) with $T=f_{2}\langle A\rangle^{-1}$, Lemma 3.7 and in addition for $T_{3}$ we apply (3.23b) with $T=f_{2}$ and (3.32a)-(3.32b). Clearly (3.28b) follows from (3.33)-(3.35) by one integration.

Re (3.28c). We mimic the proof of (3.28b). 
Next we note that the above arguments apply to $A \rightarrow A-n$ for any $n \in \mathbb{Z}$ yielding bounds being independent of $n$. Taking $\epsilon \rightarrow 0$ we thus obtain the following bounds for the accretive operator $T(z)=-\mathrm{i} f_{2} R(z, \sigma) f_{2}$, all being uniform in $n$ and in large $\sigma$ and $\nu$,

$$
\begin{gathered}
\left\|\langle A-n\rangle^{-1} T(z)\langle A-n\rangle^{-1}\right\| \leq \tilde{C}, \\
\left\|F(A<n) T(z)\langle A-n\rangle^{-2}\right\| \leq \tilde{C}, \\
\left\|\langle A-n\rangle^{-2} T(z) F(A \geq n)\right\| \leq \tilde{C} .
\end{gathered}
$$

Due to these bounds and Lemmas $3.3-3.4$ we conclude (3.16) with $C=16 \tilde{C}$ provided $\operatorname{Im} z>0$ (and hence also if $\operatorname{Im} z<0$ ).

3.4. Besov space bound of resolvent, Proposition 3.6. We introduce operators

$$
S_{\sigma}=f_{2}^{-1} f_{1} \text { and } T_{\sigma}=M\left(t_{\sigma}\right) ; t_{\sigma}(r)=\sigma\left(\frac{r}{1+r}\right)^{1 / 2}(1+r) f_{1}^{2} .
$$

Here and henceforth $M(\cdot)$ refers to the operator of multiplication by the function in the argument. We shall prove the following lemmas

Lemma 3.8. There exists $C>0$ independent of $\sigma>1$ such that

$$
\left\|S_{\sigma} v\right\|_{B(A)} \leq C\|v\|_{B\left(T_{\sigma}\right)} .
$$

Lemma 3.9. There exists $C>0$ independent of $\sigma>1$ such that

$$
\left\|f_{1}^{-1} u\right\|_{B\left(T_{\sigma}\right)} \leq C \sigma^{1 / 2}\|u\|_{B(r)} .
$$

Proof of Proposition 3.6. We combine Lemmas 3.8-3.9 to obtain that

$$
f_{2}^{-1}=S_{\sigma} f_{1}^{-1} \in \mathcal{B}(B(r), B(A))
$$

with a bounding constant of the form $C \sigma^{1 / 2}$. Whence, due to Lemma 3.5,

$$
R(z, \sigma)=f_{2}^{-1}\left(f_{2} R(z, \sigma) f_{2}\right) f_{2}^{-1} \in \mathcal{B}\left(B(r), B(r)^{*}\right)
$$

with a bounding constant of the form $C \sigma$.

Proof of Lemma 3.8. Since $\left\|S_{\sigma}\right\| \leq 1$ it suffices, due to Lemma 3.1, to show the bound

$$
\left\|A S_{\sigma} v\right\| \leq C\left(\left\|T_{\sigma} v\right\|+\|v\|\right) .
$$

Using (3.24a) we estimate for all $\psi \in \mathcal{D}(r)=\mathcal{D}(M(r))$

$$
\begin{aligned}
\left\|A f_{2}^{-1} \psi\right\|^{2} & \leq 2\left\|f_{2}^{-1} A \psi\right\|^{2}+C_{1}\left\|f_{2}^{-1} \psi\right\|^{2} \\
& \leq 4\left\|f_{2}^{-1} 2 p_{r} r \psi\right\|^{2}+C_{2}\left\|f_{2}^{-1} \psi\right\|^{2} \\
& \leq 16 \sigma^{2}\|r \psi\|^{2}+C_{2}\left\|S_{\sigma} f_{1}^{-1} \psi\right\|^{2} \\
& \leq 16 \sigma^{2}\left\|\left(\frac{r}{1+r}\right)^{1 / 2}(1+r) f_{1} \psi\right\|^{2}+C_{2}\left\|f_{1}^{-1} \psi\right\|^{2} .
\end{aligned}
$$

We apply the estimate to $\psi=f_{1} v$ yielding (3.39) with $C=\max \left(4, C_{2}^{1 / 2}\right)$. 
Proof of Lemma 3.9. Introducing $\tilde{f}_{1}=\sigma^{1 / 2} f_{1}$ we need to bound for $j=1,2,3$

$$
\begin{aligned}
\left\|\tilde{f}_{1}^{-1} F_{j} u\right\|_{B\left(T_{\sigma}\right)} & \leq C\|u\|_{B(r)} ; \\
F_{1} & =F\left(r<\sigma^{-2 / 3}\right), \\
F_{2} & =F\left(\sigma^{-2 / 3} \leq r<2\right), \\
F_{2} & =F(r \geq 2) .
\end{aligned}
$$

Using that $t_{\sigma}$ is a bounded function on the support of $F_{1}$ and (3.5) we estimate

$$
\left\|\tilde{f}_{1}^{-1} F_{1} u\right\|_{B\left(T_{\sigma}\right)} \leq C_{1} \sigma^{-1 / 6}\|u\| \leq C_{1}\|u\|_{B(r)},
$$

which agrees with (3.40).

Let $g_{\sigma}(r)=\sigma r^{3 / 2}$ and $G_{\sigma}=M\left(g_{\sigma}\right)$. Using the two-sided estimates $t_{\sigma}(r) \leq C g_{\sigma}(r)$ and $g_{\sigma}(r) \leq C t_{\sigma}(r)$, which are valid on the support of $F_{2}$, we can estimate

$$
\begin{aligned}
\left\|\tilde{f}_{1}^{-1} F_{2} u\right\|_{B\left(T_{\sigma}\right)} & \leq C\left\|(\sigma r)^{-1 / 2} F_{2} u\right\|_{B\left(G_{\sigma}\right)} \\
& =C \sum_{2 \leq j \leq J} R_{j}^{1 / 2}\left\|F\left(R_{j-1} \leq g_{\sigma}(r)<R_{j}\right)\left(\sigma r^{3 / 2}\right)^{-1 / 2} r^{1 / 4} F_{2} u\right\| \\
& \leq 2^{1 / 2} C \sum_{2 \leq j \leq J}\left\|F\left(R_{j-1} \leq g_{\sigma}(r)<R_{j}\right) r^{1 / 4} F_{2} u\right\|,
\end{aligned}
$$

where $J=J_{\sigma} \in \mathbb{N}$ is taken smallest such that $R_{J}>2^{3 / 2} \sigma$. By estimating for each term

$$
r^{1 / 4} \leq\left(R_{j} / \sigma\right)^{1 / 6} \leq 2^{(j-J+3) / 6}
$$

we thus obtain

$$
\left\|\tilde{f}_{1}^{-1} F_{2} u\right\|_{B\left(T_{\sigma}\right)} \leq 2^{1 / 2} C \sum_{2 \leq j \leq J} 2^{(j-J+3) / 6}\|u\| \leq C_{1}\|u\|_{B(r)},
$$

which also agrees with (3.40).

Finally using the two-sided estimates $t_{\sigma}(r) \leq C \sigma(1+r)$ and $\sigma(1+r) \leq C t_{\sigma}(r)$, which are valid on the support of $F_{3}$, and Lemma 3.2 we can estimate

$$
\begin{aligned}
\left\|\tilde{f}_{1}^{-1} F_{3} u\right\|_{B\left(T_{\sigma}\right)} & \leq C_{1} \sigma^{-1 / 2}\|u\|_{B(\sigma(1+r))} \\
& \leq 8 C_{1}\|u\|_{B((1+r))} \leq C_{2}\|u\|_{B(r)},
\end{aligned}
$$

which also agrees with (3.40).

Having proved (3.41a)-(3.41c) we conclude (3.40).

3.5. Case $\Omega=\mathbf{X}$. We outline a proof of the analogue of Proposition 3.6 for the case $\Omega=\mathbf{X}$. This is conceptionally simpler than the previous case, and it suffices to mimic parts of the previous proof. We can use the standard conjugate operator

$$
A=x \cdot p+p \cdot x
$$

rather than the one defined by (3.12) (alternatively $A$ is given by taking $r=|x|$ in (3.12)). We impose

$$
V(x), x \cdot \nabla V(x),(x \cdot \nabla)^{2} V(x) \in \mathcal{C}\left(H^{1}(\mathbf{X}), H^{1}(\mathbf{X})^{*}\right) .
$$


We consider a Hilbert space $\mathcal{H}=L^{2}(\mathbf{X}, \mathrm{d} x) \otimes L^{2}(M, \mathrm{~d} y)$ where interpretation of $x, y$ and $M$ is the same as in Subsection 3.2. Similarly introducing $H=\widetilde{H}^{b}+\widetilde{B}$ as before the form domains are

$$
Q\left(\widetilde{H}^{b}\right)=Q(H)=L^{2}\left(M, H^{1}(\mathbf{X}) ; \mathrm{d} y\right) \subset \mathcal{H} .
$$

Again we have the property (3.13a). We define

$$
f^{2}=1+\sigma^{-2} p^{2}, f \geq 0 .
$$

We have results similar to Lemma 3.5 and Proposition 3.6.

Lemma 3.10. With A given by (3.42) we have uniformly in all large $\sigma, \nu>1$ and all $\operatorname{Re} z \approx 1$

$$
\|f R(z, \sigma) f\|_{\mathcal{B}\left(B(A), B(A)^{*}\right)} \leq C .
$$

Proposition 3.11. We have uniformly in all large $\sigma, \nu>1$ and all $\operatorname{Re} z \approx 1$

$$
\|R(z, \sigma)\|_{\mathcal{B}\left(B(|x|), B(|x|)^{*}\right)} \leq C \sigma .
$$

Given Lemma 3.10 we notice that Proposition 3.11 is an easy consequence of the following analogues of Lemmas 3.8 and 3.9. Define

$$
T_{\sigma}=M\left(t_{\sigma}\right) ; t_{\sigma}(x, y)=\sigma(1+|x|) .
$$

Lemma 3.12. There exists $C>0$ independent of $\sigma>1$ such that

$$
\left\|f^{-1} v\right\|_{B(A)} \leq C\|v\|_{B\left(T_{\sigma}\right)} .
$$

Lemma 3.13. There exists $C>0$ independent of $\sigma>1$ such that

$$
\|u\|_{B\left(T_{\sigma}\right)} \leq C \sigma^{1 / 2}\|u\|_{B(|x|)} .
$$

We can prove Lemma 3.12 by mimicking the proof of Lemma 3.8, while Lemma 3.13 is an immediate consequence of Lemma 3.2.

Whence it remains to show Lemma 3.10. For that we note the analogue of (3.18) where now $r=|x|$

$$
\begin{aligned}
\mathrm{i}[H, A] & :=s^{-1}\left(2 p^{2}+W\right) s^{-1}+2 \operatorname{Re}\left(s^{-1}\left(\nabla r^{2} \cdot \nabla_{x} s\right) \widetilde{H}^{b}\right)-\nabla r^{2} \cdot \nabla_{x} \widetilde{B} \\
W(x) & :=-\nabla r^{2} \cdot \nabla V(x) .
\end{aligned}
$$

Using (3.48) we can indeed mimic the proof of Lemma 3.5 with $f$ replacing $f_{2}$. Note this is much simpler now. For example there are no factors of $\frac{r}{1+r}$ to consider, and the analogue of the second commutator is given by (3.27) without the second term on the right hand side. We leave the details to the reader.

\section{Appendix A}

In this appendix we show how to undo the commutator $\mathrm{i}[H, A]$. This is used to obtain (2.4). Since the Schrödinger operator $H$ is realized with the Dirichlet boundary condition the approximation procedure of [IS2] is not sufficient. 
A.1. Setting. We shall work in a generalized setting on a manifold, and present all conditions needed for the argument independently of the previous sections. The case of a constant metric is sufficient for application to (2.4). The verification of the conditions below under the conditions of Sections 1 and 2 is straightforward.

Let $(\Omega, g)$ be a Riemannian manifold of dimension $d \geq 1$, and consider the Schrödinger operator on $\mathcal{H}=L^{2}(\Omega)=L^{2}\left(\Omega,(\operatorname{det} g)^{1 / 2} \mathrm{~d} x\right)$ :

$$
H=H_{0}+V ; \quad H_{0}=-\frac{1}{2} \Delta=\frac{1}{2} p_{i}^{*} g^{i j} p_{j}, \quad p_{i}=-\mathrm{i} \partial_{i} .
$$

We realize $H_{0}$ as a self-adjoint operator by setting the Dirichlet boundary condition, i.e. $H_{0}$ is the unique self-adjoint operator associated with the closure of the quadratic form

$$
\left\langle H_{0}\right\rangle_{\psi}=\left\langle\psi,-\frac{1}{2} \Delta \psi\right\rangle, \quad \psi \in C_{\mathrm{c}}^{\infty}(\Omega) .
$$

We denote the form closure and the self-adjoint realization by the same symbol $H_{0}$. Moreover, we consider the weighted spaces

$$
\mathcal{H}^{s}=\left(H_{0}+1\right)^{-s / 2} \mathcal{H}, \quad s \in \mathbb{R},
$$

and $H_{0}$ may also be understood as $\mathcal{H}^{s} \rightarrow \mathcal{H}^{s-2}, s \in \mathbb{R}$. For the realization of $H=H_{0}+V$ we assume the following condition:

Condition A.1. The potential $V$ is a locally integrable real-valued function, and there exist $\varepsilon \in[0,1)$ and $C>0$ such that for any $\psi \in C_{\mathrm{c}}^{\infty}(\Omega)$

$$
\left|\langle V\rangle_{\psi}\right| \leq \varepsilon\left\langle H_{0}\right\rangle_{\psi}+C\|\psi\|^{2}
$$

By this condition we extend the form domain of $V$ as $Q(V)=\mathcal{H}^{1}$, and this defines a bounded operator $V: \mathcal{H}^{1} \rightarrow \mathcal{H}^{-1}$. We note, though, this quadratic form is not necessarily closed. We henceforth consider $H=H_{0}+V$ as a closed quadratic form on $Q(H)=\mathcal{H}^{1}$ or, equivalently, as a bounded operator $\mathcal{H}^{1} \rightarrow \mathcal{H}^{-1}$. Then the Friedrichs self-adjoint realization of $H$ on $\mathcal{H}$ is the restriction of this $H: \mathcal{H}^{1} \rightarrow \mathcal{H}^{-1}$ to the domain:

$$
\mathcal{D}(H)=\left\{\psi \in \mathcal{H}^{1} \mid H \psi \in \mathcal{H}\right\} \subset \mathcal{H} .
$$

We next assume a regularity condition for the (virtual) boundary of $\Omega$ :

Condition A.2. There exists a real-valued function $r \in C^{\infty}(\Omega)$ such that:

(1) The gradient vector field $2 \omega=\operatorname{grad} r^{2}$ on $\Omega$ is complete.

(2) The following bounds hold:

$$
\sup |\mathrm{d} r|<\infty, \quad \sup \left|\nabla^{2} r^{2}\right|<\infty, \quad \sup _{r \rightarrow \infty}\left|\mathrm{d} \Delta r^{2}\right|<\infty .
$$

The function $r$ of Condition A.2 is a generalization of that of previous sections. For the $r$ of Sections 1 and 2 we refer to Lemma 1.2 and Subsection 1.3 (the completeness is valid because the vector field $\omega$ is tangent to the boundary $\partial \Omega$ ). For the $r$ of Subsection 3.2 we refer to $(3.11 b)$ (the completeness is valid because $\omega$ vanishes at the boundary $\partial \Omega \times M)$. For the $r$ of Subsection 3.5 the properties (1) and (2) are obvious, however there is a cusp singularity at $x=0$ in this case. A substitute for Lemmas A.7-A.9, shown under Conditions A.1-A.2, is in this case immedidately provided by the formula $\left\|p \mathrm{e}^{\mathrm{i} t A} \psi\right\|=\mathrm{e}^{2 t}\|p \psi\|$. 
By Condition A.2 (1) the vector field $\operatorname{grad} r^{2}$ generates a one-parameter group of diffeomorphisms on $\Omega$, which we denote by

$$
\mathrm{e}^{2 \cdot} \cdot: \mathbb{R} \times \Omega \rightarrow \Omega, \quad(t, x) \mapsto \mathrm{e}^{2 t} x .
$$

This satisfies by definition, in local coordinates,

$$
\partial_{t}\left(\mathrm{e}^{2 t} x\right)^{i}=g^{i j}\left(\mathrm{e}^{2 t} x\right)\left(\partial_{j} r^{2}\right)\left(\mathrm{e}^{2 t} x\right)
$$

We define the dilation $\mathrm{e}^{\mathrm{i} t A}: \mathcal{H} \rightarrow \mathcal{H}$ with respect to $r$ by the one-parameter unitary group

$$
\mathrm{e}^{\mathrm{i} t A} u(x)=J\left(\mathrm{e}^{2 t} ; x\right)^{1 / 2}\left(\frac{\operatorname{det} g\left(\mathrm{e}^{2 t} x\right)}{\operatorname{det} g(x)}\right)^{1 / 4} u\left(\mathrm{e}^{2 t} x\right),
$$

where $J$ is the relevant Jacobian. Note that there is another expression:

$$
\mathrm{e}^{\mathrm{i} t A} u(x)=\exp \left(\int_{0}^{t} \frac{1}{2}\left(\Delta r^{2}\right)\left(\mathrm{e}^{2 s} x\right) \mathrm{d} s\right) u\left(\mathrm{e}^{2 t} x\right) .
$$

We let $A$ be the generator of $\mathrm{e}^{\mathrm{i} t A}$. By the unitarity of $\mathrm{e}^{\mathrm{i} t A}$ the operator $A$ is selfadjoint, and $C_{\mathrm{c}}^{\infty}(\Omega) \subseteq \mathcal{D}(A)$ is a core for it. In fact, the dense subspace $C_{\mathrm{c}}^{\infty}(\Omega) \subseteq \mathcal{H}$ is invariant under $\mathrm{e}^{\mathrm{i} t \bar{A}}$, and for any $u \in C_{\mathrm{c}}^{\infty}(\Omega)$ the limit

$$
\lim _{t \rightarrow 0} t^{-1}\left(\mathrm{e}^{\mathrm{i} t A} u-u\right)
$$

exists in $\mathcal{H}$. Note that by $(\mathrm{A} .4)$ we have $A$ on $C_{\mathrm{c}}^{\infty}(\Omega)$ written by

$$
A=\mathrm{i}\left[H_{0}, r^{2}\right]=\frac{1}{2}\left\{\left(\partial_{i} r^{2}\right) g^{i j} p_{j}+p_{i}^{*} g^{i j}\left(\partial_{j} r^{2}\right)\right\}=r p^{r}+\left(p^{r}\right)^{*} r,
$$

where $p^{r}=-\mathrm{i} \partial^{r}=-\mathrm{i}\left(\partial_{i} r\right) g^{i j} \partial_{j}$.

Let us first consider the commutator $\mathrm{i}[H, A]$ as a quadratic form defined for $\psi \in$ $C_{\mathrm{c}}^{\infty}(\Omega)$ by

$$
\langle\mathrm{i}[H, A]\rangle_{\psi}=\mathrm{i}\langle H \psi, A \psi\rangle-\mathrm{i}\langle A \psi, H \psi\rangle .
$$

In order to discuss its extension we impose the following abstract form bound condition, which is not quite independent of Conditions A.1 and A.2.

Condition A.3. There exists $C>0$ such that for any $\psi \in C_{\mathrm{c}}^{\infty}(\Omega)$

$$
\left|\langle\mathrm{i}[H, A]\rangle_{\psi}\right| \leq C\left\langle H_{0}+1\right\rangle_{\psi} \text {. }
$$

Similarly to the above, we henceforth regard $\mathrm{i}[H, A]$ as a quadratic form on $Q(\mathrm{i}[H, A])=\mathcal{H}^{1}$, which may not be closed, or as a bounded operator $\mathcal{H}^{1} \rightarrow \mathcal{H}^{-1}$.

A.2. Preliminaries. We prove a regularity property of the flow (A.2).

Lemma A.4. There exists $C>0$ such that for any $t \in \mathbb{R}$ and $x \in \Omega$

$$
d \mathrm{e}^{-C|t|} \leq g^{i j}(x) g_{k l}\left(\mathrm{e}^{2 t} x\right)\left[\partial_{i}\left(\mathrm{e}^{2 t} x\right)^{k}\right]\left[\partial_{j}\left(\mathrm{e}^{2 t} x\right)^{l}\right] \leq d \mathrm{e}^{C|t|} .
$$

Proof. The proof is similar to that of [IS1, Lemma 2.3]. We note that the expression in the middle of (A.5) is independent of choice of coordinates. Fix $x \in \Omega$ and choose coordinates such that $g_{i j}(x)=\delta_{i j}$. Consider the vector fields along $\left\{\mathrm{e}^{2 t} x\right\}_{t \in \mathbb{R}}$ given by $\partial_{i} \mathrm{e}^{2 t} x$ and $\partial_{j} \mathrm{e}^{2 t} x$. Since the Levi-Civita connection $\nabla$ is compatible with the metric,

$$
\begin{aligned}
\frac{\partial}{\partial t} g_{k l}\left(\mathrm{e}^{2 t} x\right) & {\left[\partial_{i}\left(\mathrm{e}^{2 t} x\right)^{k}\right]\left[\partial_{j}\left(\mathrm{e}^{2 t} x\right)^{l}\right]=\frac{\partial}{\partial t}\left\langle\partial_{i} \mathrm{e}^{2 t} x, \partial_{j} \mathrm{e}^{2 t} x\right\rangle } \\
& =\left\langle\nabla_{\partial_{t} \mathrm{e}^{2 t} x} \partial_{i} \mathrm{e}^{2 t} x, \partial_{j} \mathrm{e}^{2 t} x\right\rangle+\left\langle\partial_{i} \mathrm{e}^{2 t} x, \nabla_{\partial_{t} \mathrm{e}^{2 t} x} \partial_{j} \mathrm{e}^{2 t} x\right\rangle .
\end{aligned}
$$


(The definition of $\nabla_{\partial_{t} \mathrm{e}^{2 t} x}$ is given below.) From (A.3) it follows that

$$
\begin{aligned}
\nabla_{\partial_{t} \mathrm{e}^{2 t} x} \partial_{i}\left(\mathrm{e}^{2 t} x\right)^{\bullet} & =\partial_{t} \partial_{i}\left(\mathrm{e}^{2 t} x\right)^{\bullet}+\left[\partial_{t}\left(\mathrm{e}^{2 t} x\right)^{k}\right] \Gamma_{k l}^{\bullet} \partial_{i}\left(\mathrm{e}^{2 t} x\right)^{l} \\
& =\partial_{i} \partial_{t}\left(\mathrm{e}^{2 t} x\right)^{\bullet}+\left(g^{k m} \partial_{m} r^{2}\right) \Gamma_{k l}^{\bullet} \partial_{i}\left(\mathrm{e}^{2 t} x\right)^{l} \\
& =\left[\partial_{i}\left(\mathrm{e}^{2 t} x\right)^{k}\right] \partial_{k}\left(g^{\bullet l} \partial_{l} r^{2}\right)+\left[\partial_{i}\left(\mathrm{e}^{2 t} x\right)^{l}\right] \Gamma_{k l}^{\bullet} g^{k m} \partial_{m} r^{2} \\
& =\nabla_{\partial_{i} \mathrm{e}^{2 t} x}\left(g^{\bullet l} \partial_{l} r^{2}\right) \\
& =g^{\bullet l}\left[\partial_{i}\left(\mathrm{e}^{2 t} x\right)^{k}\right]\left(\nabla^{2} r^{2}\right)_{k l} .
\end{aligned}
$$

Thus, plugging this into (A.6) and taking a contraction with $g^{i j}(x)=\delta^{i j}$, we obtain

$$
\left|\frac{\partial}{\partial t} g^{i j}(x) g_{k l}\left(\mathrm{e}^{2 t} x\right)\left[\partial_{i}\left(\mathrm{e}^{2 t} x\right)^{k}\right]\left[\partial_{j}\left(\mathrm{e}^{2 t} x\right)^{l}\right]\right| \leq C g^{i j}(x) g_{k l}\left(\mathrm{e}^{2 t} x\right)\left[\partial_{i}\left(\mathrm{e}^{2 t} x\right)^{k}\right]\left[\partial_{j}\left(\mathrm{e}^{2 t} x\right)^{l}\right] .
$$

Noting $\left.g^{i j}(x) g_{k l}\left(\mathrm{e}^{2 t} x\right)\left[\partial_{i}\left(\mathrm{e}^{2 t} x\right)^{k}\right]\left[\partial_{j}\left(\mathrm{e}^{2 t} x\right)^{l}\right]\right|_{t=0}=d$, we have (A.5).

Recall the functions $\chi_{\nu}, \bar{\chi}_{\nu} \in C^{\infty}(\mathbb{R})$ of Subsubsection 1.3.3. We shall henceforth consider the functions $\chi_{\nu}=\chi_{\nu}(r), \bar{\chi}_{\nu}=\bar{\chi}_{\nu}(r)$ as being composed with the function $r$ from Condition A.2. We also set

$$
\chi_{\nu, \nu^{\prime}}=\chi_{\nu} \bar{\chi}_{\nu^{\prime}}, \quad \bar{\chi}_{\nu^{\prime}}=1-\chi_{\nu^{\prime}}, \quad \nu^{\prime} \geq 2 \nu \geq 2 .
$$

Next, we prove the following statement:

Lemma A.5. Let $\psi \in \mathcal{D}(H)$. Then there exists $\nu_{0}>0$ such that, for any $\nu>\nu_{0}$ and any $\sigma \geq 0$ with $\mathrm{e}^{\sigma r} \psi, \mathrm{e}^{\sigma r} H \psi \in \mathcal{H}$, one has $\mathrm{e}^{\sigma r} \chi_{\nu} \psi \in \mathcal{D}(H)$.

Proof. Step I. We first claim $\mathrm{e}^{\sigma r} \chi_{\nu, \nu^{\prime}} \psi \in \mathcal{D}(H)$. Since $\psi \in \mathcal{H}^{1}$, we have

$$
\mathrm{e}^{\sigma r} \chi_{\nu, \nu^{\prime}} \psi, \mathrm{e}^{\sigma r} \chi_{\nu, \nu^{\prime}} p \psi \in \mathcal{H}
$$

and hence $p \mathrm{e}^{\sigma r} \chi_{\nu, \nu^{\prime}} \psi \in \mathcal{H}$ by (A.1). Choose a sequence $\psi_{n} \in C_{\mathrm{c}}^{\infty}(\Omega)$ such that, as $n \rightarrow \infty$,

$$
\left\|\psi-\psi_{n}\right\|+\left\|p\left(\psi-\psi_{n}\right)\right\| \rightarrow 0
$$

and then by (A.1) again, as $n \rightarrow \infty$,

$$
\mathrm{e}^{\sigma r} \chi_{\nu, \nu^{\prime}} \psi_{n} \rightarrow \mathrm{e}^{\sigma r} \chi_{\nu, \nu^{\prime}} \psi, \quad p \mathrm{e}^{\sigma r} \chi_{\nu, \nu^{\prime}} \psi_{n} \rightarrow p \mathrm{e}^{\sigma r} \chi_{\nu, \nu^{\prime}} \psi \quad \text { in } \mathcal{H}
$$

This implies that $\mathrm{e}^{\sigma r} \chi_{\nu, \nu^{\prime}} \psi \in \mathcal{H}^{1}$. Note the distributional identity

$$
H \mathrm{e}^{\sigma r} \chi_{\nu, \nu^{\prime}} \psi=\mathrm{e}^{\sigma r} \chi_{\nu, \nu^{\prime}} H \psi-\mathrm{e}^{\sigma r}\left(\sigma \chi_{\nu, \nu^{\prime}}+\chi_{\nu, \nu^{\prime}}^{\prime}\right) \partial^{r} \psi-\frac{1}{2}\left(\Delta \mathrm{e}^{\sigma r} \chi_{\nu, \nu^{\prime}}\right) \psi .
$$

Then, since $\psi, p \psi, H \psi \in \mathcal{H}$ and by (A.1)

$$
\chi_{\nu}|\Delta r|=\left.\frac{1}{2 r} \chi_{\nu}\left|\left(\Delta r^{2}\right)-2\right| \mathrm{d} r\right|^{2} \mid \leq C_{\nu},
$$

we have $H \mathrm{e}^{\sigma r} \chi_{\nu, \nu^{\prime}} \psi \in \mathcal{H}$. Hence $\mathrm{e}^{\sigma r} \chi_{\nu, \nu^{\prime}} \psi \in \mathcal{D}(H)$.

Step II. We next show $\mathrm{e}^{\sigma r} \chi_{\nu} p \psi \in \mathcal{H}$. Noting that $\mathrm{e}^{\sigma r} \chi_{\nu, \nu^{\prime}} \psi \in \mathcal{H}^{1}$ as in Step I, we estimate by Condition A.1

$$
\begin{aligned}
\left\|\mathrm{e}^{\sigma r} \chi_{\nu, \nu^{\prime}} p \psi\right\|^{2} & \leq 2\left\|p \mathrm{e}^{\sigma r} \chi_{\nu, \nu^{\prime}} \psi\right\|^{2}+2\left\|\left(p \mathrm{e}^{\sigma r} \chi_{\nu, \nu^{\prime}}\right) \psi\right\|^{2} \\
& \leq C\langle H\rangle_{\mathrm{e}^{\sigma r} \chi_{\nu, \nu^{\prime}} \psi}+C_{\sigma}\left\|\mathrm{e}^{\sigma r} \chi_{\nu / 2,2 \nu^{\prime}} \psi\right\|^{2} .
\end{aligned}
$$


Then, by $\mathrm{e}^{\sigma r} \chi_{\nu, \nu^{\prime}} \psi \in \mathcal{D}(H)$ and the definition of the Friedrichs extension

$$
\begin{aligned}
\left\|\mathrm{e}^{\sigma r} \chi_{\nu, \nu^{\prime}} p \psi\right\|^{2} \leq & C\left\langle\mathrm{e}^{\sigma r} \chi_{\nu, \nu^{\prime}} \psi, H \mathrm{e}^{\sigma r} \chi_{\nu, \nu^{\prime}} \psi\right\rangle+C_{\sigma}\left\|\mathrm{e}^{\sigma r} \chi_{\nu / 2,2 \nu^{\prime}} \psi\right\|^{2} \\
\leq & C\left\langle\mathrm{e}^{\sigma r} \chi_{\nu, \nu^{\prime}} \psi, \mathrm{e}^{\sigma r} \chi_{\nu, \nu^{\prime}} H \psi\right\rangle+C\left\langle\mathrm{e}^{\sigma r} \chi_{\nu, \nu^{\prime}} \psi,\left[-\frac{1}{2} \Delta, \mathrm{e}^{\sigma r} \chi_{\nu, \nu^{\prime}}\right] \psi\right\rangle \\
& +C_{\sigma}\left\|\mathrm{e}^{\sigma r} \chi_{\nu / 2,2 \nu^{\prime}} \psi\right\|^{2} \\
\leq & C\left\|\mathrm{e}^{\sigma r} \chi_{\nu, \nu^{\prime}} H \psi\right\|^{2}+\frac{1}{2}\left\|\mathrm{e}^{\sigma r} \chi_{\nu, \nu^{\prime}} p \psi\right\|^{2}+C_{\sigma}\left\|\mathrm{e}^{\sigma r} \chi_{\nu / 2,2 \nu^{\prime}} \psi\right\|^{2} .
\end{aligned}
$$

Hence

$$
\left\|\mathrm{e}^{\sigma r} \chi_{\nu, \nu^{\prime}} p \psi\right\|^{2} \leq C\left\|\mathrm{e}^{\sigma r} \chi_{\nu, \nu^{\prime}} H \psi\right\|^{2}+C_{\sigma}\left\|\mathrm{e}^{\sigma r} \chi_{\nu / 2,2 \nu^{\prime}} \psi\right\|^{2} .
$$

Now we let $\nu^{\prime} \rightarrow \infty$. Then by the Lebesgue convergence theorem $\mathrm{e}^{\sigma r} \chi_{\nu} p \psi \in \mathcal{H}$.

Step III. We note $p \mathrm{e}^{\sigma r} \chi_{\nu} \psi \in \mathcal{H}$ by Step II. We choose a sequence $\psi_{n} \in C_{\mathrm{c}}^{\infty}(\Omega)$ satisfying (A.7) as $n \rightarrow \infty$, and estimate

$$
\left\|\mathrm{e}^{\sigma r} \chi_{\nu} \psi-\mathrm{e}^{\sigma r} \chi_{\nu, \nu^{\prime}} \psi_{n}\right\|+\left\|p\left(\mathrm{e}^{\sigma r} \chi_{\nu} \psi-\mathrm{e}^{\sigma r} \chi_{\nu, \nu^{\prime}} \psi_{n}\right)\right\| .
$$

For $\nu^{\prime} \geq 2 \nu$ we have the first term of (A.9) bounded by

$$
\left\|\mathrm{e}^{\sigma r} \chi_{\nu} \psi-\mathrm{e}^{\sigma r} \chi_{\nu, \nu^{\prime}} \psi_{n}\right\| \leq\left\|\mathrm{e}^{\sigma r} \chi_{\nu^{\prime}} \psi\right\|+\left\|\mathrm{e}^{\sigma r} \chi_{\nu, \nu^{\prime}}\left(\psi-\psi_{n}\right)\right\|
$$

and the second term bounded by

$$
\begin{aligned}
& \left\|p\left(\mathrm{e}^{\sigma r} \chi_{\nu} \psi-\mathrm{e}^{\sigma r} \chi_{\nu, \nu^{\prime}} \psi_{n}\right)\right\| \\
& \leq\left\|p \mathrm{e}^{\sigma r} \chi_{\nu^{\prime}} \psi\right\|+\left\|p \mathrm{e}^{\sigma r} \chi_{\nu, \nu^{\prime}}\left(\psi-\psi_{n}\right)\right\| \\
& \leq\left\|\mathrm{e}^{\sigma r} \chi_{\nu^{\prime}} p \psi\right\|+C_{\sigma}\left\|\mathrm{e}^{\sigma r} \chi_{\nu^{\prime} / 2,2 \nu^{\prime}} \psi\right\|+\left\|\mathrm{e}^{\sigma r} \chi_{\nu, \nu^{\prime}} p\left(\psi-\psi_{n}\right)\right\|+C_{\sigma}\left\|\mathrm{e}^{\sigma r} \chi_{\nu^{\prime} / 2,2 \nu^{\prime}}\left(\psi-\psi_{n}\right)\right\| .
\end{aligned}
$$

Thus we can make (A.9) arbitrarily small by letting $\nu^{\prime}$ be large and then $n$ large. Then we obtain a sequence verifying

$$
\left\|\mathrm{e}^{\sigma r} \chi_{\nu} \psi-\mathrm{e}^{\sigma r} \chi_{\nu, \nu^{\prime}(m)} \psi_{n(m)}\right\|+\left\|p\left(\mathrm{e}^{\sigma r} \chi_{\nu} \psi-\mathrm{e}^{\sigma r} \chi_{\nu, \nu^{\prime}(m)} \psi_{n(m)}\right)\right\| \rightarrow 0
$$

as $m \rightarrow \infty$, and hence $\mathrm{e}^{\sigma r} \chi_{\nu} \psi \in \mathcal{H}^{1}$.

Finally, noting the distributional identity

$$
H \mathrm{e}^{\sigma r} \chi_{\nu} \psi=\mathrm{e}^{\sigma r} \chi_{\nu} H \psi+\left[-\frac{1}{2} \Delta, \mathrm{e}^{\sigma r} \chi_{\nu}\right] \psi,
$$

we learn $H \mathrm{e}^{\sigma r} \chi_{\nu} \psi \in \mathcal{H}$, and hence $\mathrm{e}^{\sigma r} \chi_{\nu} \psi \in \mathcal{D}(H)$.

Corollary A.6. Let $\psi \in \mathcal{D}(H)$ satisfy $\mathrm{e}^{\sigma r} \psi, \mathrm{e}^{\sigma r} H \psi \in \mathcal{H}$ for any $\sigma \geq 0$. Then for large $\nu>0$ one has $\mathrm{e}^{\sigma r} \chi_{\nu} \psi \in \mathcal{D}(H) \cap \mathcal{D}(A)$.

\section{A.3. Undoing commutators.}

Lemma A.7. For any $s \in[-1,1]$ the inclusion $\mathrm{e}^{\mathrm{i} t A} \mathcal{H}^{s} \subseteq \mathcal{H}^{s}$ holds, and

$$
\sup _{|t|<1}\left\|\mathrm{e}^{\mathrm{i} t A}\right\|_{\mathcal{B}\left(\mathcal{H}^{s}\right)}<\infty \text {. }
$$

Moreover, $\mathrm{e}^{\mathrm{i} t A}: \mathcal{H}^{s} \rightarrow \mathcal{H}^{s}$ is strongly continuous in $t \in \mathbb{R}$.

Proof. Let us first set $s=1$. For any $\psi \in C_{\mathrm{c}}^{\infty}(\Omega)$ we can compute by (A.4)

$$
\begin{aligned}
& p_{i}\left(\mathrm{e}^{\mathrm{i} t A} \psi\right)(x) \\
& =\left(\int_{0}^{t} \frac{1}{2}\left[p_{i}\left(\mathrm{e}^{2 s} x\right)^{j}\right]\left(\partial_{j} \Delta r^{2}\right)\left(\mathrm{e}^{2 s} x\right) \mathrm{d} s\right)\left(\mathrm{e}^{\mathrm{i} t A} \psi\right)(x)+\left[\partial_{i}\left(\mathrm{e}^{2 t} x\right)^{j}\right]\left(\mathrm{e}^{\mathrm{i} t A} p_{j} \psi\right)(x),
\end{aligned}
$$


where we abuse $\mathrm{e}^{\mathrm{i} t A}$ as if it were defined for the sections of the cotangent bundle. Then by (A.1) and Lemma A.4 for any $|t| \leq T$

$$
\begin{aligned}
\left\|\mathrm{e}^{\mathrm{i} t A} \psi\right\|_{\mathcal{H}^{1}}^{2} & =\|\psi\|_{\mathcal{H}}^{2}+\left\|p \mathrm{e}^{\mathrm{i} t A} \psi\right\|_{\mathcal{H}}^{2} \\
& \leq\|\psi\|_{\mathcal{H}}^{2}+C_{T}\left\|\mathrm{e}^{\mathrm{i} t A} \psi\right\|_{\mathcal{H}}^{2}+C_{T}\left\|\mathrm{e}^{\mathrm{i} t A} p \psi\right\|_{\mathcal{H}}^{2} \\
& \leq C_{T}\|\psi\|_{\mathcal{H}^{1}}^{2} .
\end{aligned}
$$

By the density argument this implies $\mathrm{e}^{\mathrm{i} t A} \mathcal{H}^{1} \subseteq \mathcal{H}^{1}$, and moreover for any $\psi \in \mathcal{H}^{1}$ and $|t| \leq T$

$$
\left\|\mathrm{e}^{\mathrm{i} t A} \psi\right\|_{\mathcal{H}^{1}}^{2} \leq C_{T}\|\psi\|_{\mathcal{H}^{1}}^{2} .
$$

Thus the uniform boundedness principle applies and (A.10) follows for $s=1$. As for the strong continuity as $\mathcal{H}^{1} \rightarrow \mathcal{H}^{1}$, we can show it first on $C_{\mathrm{c}}^{\infty}(\Omega)$ using (A.11) and $\partial_{i}\left(\mathrm{e}^{2 t} x\right)^{j}=\delta_{i}^{j}$, and then extend it by the boundedness. We can show the same result for $s=-1$ by taking the adjoint, and then the assertion is proved for $s \in[-1,1]$ by interpolation.

Lemma A.8. There exists $C>0$ such that for any $|t|<1$

$$
\left\|H \mathrm{e}^{\mathrm{i} t A}-\mathrm{e}^{\mathrm{i} t A} H\right\|_{\mathcal{B}\left(\mathcal{H}^{1}, \mathcal{H}^{-1}\right)} \leq C|t|
$$

Proof. As a quadratic form on $C_{\mathrm{c}}^{\infty}(\Omega)$, or as an operator $C_{\mathrm{c}}^{\infty}(\Omega) \rightarrow \mathcal{H}^{-1}$,

$$
\begin{aligned}
H \mathrm{e}^{\mathrm{i} t A}-\mathrm{e}^{\mathrm{i} t A} H & =\int_{0}^{t} \frac{\mathrm{d}}{\mathrm{d} s} \mathrm{e}^{\mathrm{i}(t-s) A} H \mathrm{e}^{\mathrm{i} s A} \mathrm{~d} s \\
& =\int_{0}^{t} \mathrm{e}^{\mathrm{i} s A \mathrm{i}[H, A] \mathrm{e}^{\mathrm{i}(t-s) A} \mathrm{~d} s .}
\end{aligned}
$$

Then by Lemma A.7 and the density argument of $C_{\mathrm{c}}^{\infty}(\Omega) \subseteq \mathcal{H}^{1}$ the assertion follows.

Lemma A.9. The following strong limit to the right exists in $\mathcal{B}\left(\mathcal{H}^{1}, \mathcal{H}^{-1}\right)$, and the following equality holds

$$
\mathrm{i}[H, A]=\mathrm{s}_{t \rightarrow 0}^{-} \lim ^{-1}\left[H \mathrm{e}^{\mathrm{i} t A}-\mathrm{e}^{\mathrm{i} t A} H\right] .
$$

Proof. For any $\psi \in C_{\mathrm{c}}^{\infty}(\Omega)$

$$
t^{-1}\left(H \mathrm{e}^{\mathrm{i} t A}-\mathrm{e}^{-\mathrm{i} t A} H\right) \psi-\mathrm{i}[H, A] \psi=t^{-1} \int_{0}^{t}\left\{\mathrm{e}^{\mathrm{i} s A} \mathrm{i}[H, A] \mathrm{e}^{\mathrm{i}(t-s) A}-\mathrm{i}[H, A]\right\} \psi \mathrm{d} s .
$$

We use the strong continuity of $\mathrm{e}^{\mathrm{i} t A}$ of Lemma A.7 to obtain (A.12) on $C_{\mathrm{c}}^{\infty}(\Omega)$. Then by Lemma A.8 and the density argument, the strong limit of (A.12) exists in $\mathcal{B}\left(\mathcal{H}^{1}, \mathcal{H}^{-1}\right)$.

The following lemma is a main goal of this appendix:

Lemma A.10. Let $\psi \in \mathcal{D}(H)$ satisfy $\mathrm{e}^{\sigma r} \psi, \mathrm{e}^{\sigma r} H \psi \in \mathcal{H}$ for any $\sigma \geq 0$. Then,

$$
\langle\mathrm{i}[H, A]\rangle_{\mathrm{e}^{\sigma r} \chi_{\nu} \psi}=\mathrm{i}\left\langle H \mathrm{e}^{\sigma r} \chi_{\nu} \psi, A \mathrm{e}^{\sigma r} \chi_{\nu} \psi\right\rangle-\mathrm{i}\left\langle A \mathrm{e}^{\sigma r} \chi_{\nu} \psi, H \mathrm{e}^{\sigma r} \chi_{\nu} \psi\right\rangle .
$$

Proof. We note $\mathrm{e}^{\sigma r} \chi_{\nu} \psi \in \mathcal{D}(H) \cap \mathcal{D}(A)$ by Corollary A.6. Then, by Lemma A.9

$$
\begin{aligned}
\langle\mathrm{i}[H, A]\rangle_{\mathrm{e}^{\sigma r} \chi_{\nu} \psi} & =\lim _{t \rightarrow 0}\left\langle t^{-1}\left[H \mathrm{e}^{\mathrm{i} t A}-\mathrm{e}^{\mathrm{i} t A} H\right]\right\rangle_{\mathrm{e}^{\sigma r}} \chi_{\nu} \psi \\
& =\mathrm{i}\left\langle H \mathrm{e}^{\sigma r} \chi_{\nu} \psi, A \mathrm{e}^{\sigma r} \chi_{\nu} \psi\right\rangle-\mathrm{i}\left\langle A \mathrm{e}^{\sigma r} \chi_{\nu} \psi, H \mathrm{e}^{\sigma r} \chi_{\nu} \psi\right\rangle .
\end{aligned}
$$


Finally we examine (3.13a) and (3.13b). It is easy to prove (3.13a) by Lemma A.7 for $s=1$ combined with the smallness of $V$, cf. Condition A.1. The bound (3.13b) is already obtained by the explicit formula $\left\|p_{r} \mathrm{e}^{\mathrm{i} t A} \psi\right\|=\mathrm{e}^{2 t}\left\|p_{r} \psi\right\|$, but we show the corresponding slightly general statement under the setting of the appendix for the quadratic form $P_{r}:=\left\{p^{r}+\left(p^{r}\right)^{*}\right\}^{2}$. We consider $P_{r}$ as a closed form with domain $Q\left(P_{r}\right)=\mathcal{D}\left(p^{r}\right)$.

Lemma A.11. The inclusion $\mathrm{e}^{\mathrm{i} t A} Q\left(P_{r}\right) \subseteq Q\left(P_{r}\right)$ holds, and

$$
\sup _{|t|<1}\left\|\mathrm{e}^{\mathrm{i} t A}\right\|_{\mathcal{B}\left(Q\left(P_{r}\right)\right)}<\infty \text {. }
$$

Proof. We first note that

$$
\partial_{t} r\left(\mathrm{e}^{2 t} x\right)=2 r\left(\mathrm{e}^{2 t} x\right)\left(\partial^{r} r\right)\left(\mathrm{e}^{2 t} x\right)=2 r\left(\mathrm{e}^{2 t} x\right)\left|\mathrm{d} r\left(\mathrm{e}^{2 t} x\right)\right|^{2}
$$

and this implies

$$
r\left(\mathrm{e}^{2 t} x\right)=r(x) \exp \left(2 \int_{0}^{t}\left|\mathrm{~d} r\left(\mathrm{e}^{2 s} x\right)\right|^{2} \mathrm{~d} s\right) .
$$

Let $\psi \in C_{\mathrm{c}}^{\infty}(M)$. Then, by $A \mathrm{e}^{\mathrm{i} t A} \psi=\mathrm{e}^{\mathrm{i} t A} A \psi$ and $A=r\left(p^{r}+\left(p^{r}\right)^{*}\right)+\frac{1}{\mathrm{i}}|\mathrm{d} r|^{2}$, we can compute

$$
\begin{aligned}
& r(x)\left(\left(p^{r}+\left(p^{r}\right)^{*}\right) \mathrm{e}^{\mathrm{i} t A} \psi\right)(x) \\
& =\left(\mathrm{e}^{\mathrm{i} t A} r\left(p^{r}+\left(p^{r}\right)^{*}\right) \psi\right)(x)+\frac{1}{\mathrm{i}}\left(\left|\mathrm{d} r\left(\mathrm{e}^{2 t} x\right)\right|^{2}-|\mathrm{d} r(x)|^{2}\right)\left(\mathrm{e}^{\mathrm{i} t A} \psi\right)(x) \\
& =r\left(\mathrm{e}^{2 t} x\right)\left(\mathrm{e}^{\mathrm{i} t A}\left(p^{r}+\left(p^{r}\right)^{*}\right) \psi\right)(x)+\frac{1}{\mathrm{i}}\left[\int_{0}^{t} \partial_{s}\left|\mathrm{~d} r\left(\mathrm{e}^{2 s} x\right)\right|^{2} \mathrm{~d} s\right]\left(\mathrm{e}^{\mathrm{i} t A} \psi\right)(x) \\
& =r\left(\mathrm{e}^{2 t} x\right)\left(\mathrm{e}^{\mathrm{i} t A}\left(p^{r}+\left(p^{r}\right)^{*}\right) \psi\right)(x)+\frac{2}{\mathrm{i}}\left[\int_{0}^{t} r\left(\mathrm{e}^{2 s} x\right)\left(\partial^{r}|\mathrm{~d} r|^{2}\right)\left(\mathrm{e}^{2 s} x\right) \mathrm{d} s\right]\left(\mathrm{e}^{\mathrm{i} t A} \psi\right)(x),
\end{aligned}
$$

so that we obtain using (A.14)

$$
\left|\left(\left(p^{r}+\left(p^{r}\right)^{*}\right) \mathrm{e}^{\mathrm{i} t A} \psi\right)(x)\right| \leq C_{T}\left[\left|\left(\mathrm{e}^{\mathrm{i} t A}\left(p^{r}+\left(p^{r}\right)^{*}\right) \psi\right)(x)\right|+\left|\left(\mathrm{e}^{\mathrm{i} t A} \psi\right)(x)\right|\right]
$$

for $|t|<T$ and $x \notin r^{-1}(0)$. For $x \in r^{-1}(0)$ it is obvious that $\mathrm{e}^{2 t} x=x$, and hence (A.15) holds also in the interior of the closed set $r^{-1}(0)$. Since both sides of (A.15) are continuous, we have (A.15) in $\Omega$ and it follows that

$$
\left\|\mathrm{e}^{\mathrm{i} t A} \psi\right\|_{Q\left(P_{r}\right)}^{2} \leq C_{T}\|\psi\|_{Q\left(P_{r}\right)}^{2}
$$

By the density argument and the uniform boundedness theorem the assertion follows.

\section{APPENDIX B}

In this appendix we introduce the notion of strictly convexity of an obstacle and derive the geometric properties needed for the one-body type model considered in Subsection 3.2.

Let $\Theta \subset \mathbb{R}^{d}, d \geq 2$, be a bounded open set, denote its closure by $\bar{\Theta}$, and set $\Omega=\mathbb{R}^{d} \backslash \bar{\Theta}$. The goal of these short notes is to give a criterion for the existence of a function $r \in C^{\infty}(\Omega)$ such that for some $c>0$

$$
\begin{gathered}
|\nabla r|=1 \text { in } \Omega, \\
\left(\nabla^{2} r\right)_{\mid S_{r}} \geq c\langle r\rangle^{-1} g_{\mid S_{r}}, \\
\left|\partial^{\alpha} r\right| \leq C_{\alpha}\langle r\rangle^{1-|\alpha|},
\end{gathered}
$$


where $g$ is the Euclidean metric, $S_{r}=r^{-1}(r)$ is the level surface and $g_{\mid S_{r}}$ is the pullback of $g$ to $S_{r}$. Note that $S_{r}$ is smooth by (B.1a). We impose the following convexity type condition for $\Theta$. Note that the inequality (B.1b) represents the convexity of $r$.

Condition B.1. Let $\Theta \subset \mathbb{R}^{d}, d \geq 2$, be an open connected subset with smooth boundary $S=\partial \Theta$, and $\nu \in \Gamma\left(N^{+} \bar{S}\right)$ be the outward unit normal vector field on $S$. There exists a constant $\kappa>0$ such that

$$
(\nabla \nu)_{\mid S} \geq \kappa g_{\mid S} .
$$

A subset $\Theta \subset \mathbb{R}^{d}$ fulfilling Condition B.1 is called strictly convex. We show in Lemma B.5 that such set is convex.

Remarks B.2. (1) The symmetric tensor $(\nabla \nu)_{\mid S}$ is called the second fundamental form of $S$, and its eigenvalues relative to $g_{\mid S}$ are called the principal curvatures. Hence (B.2) implies that the principal curvatures are bounded below by $\kappa>0$. For these notions we refer to [Ch, Section II.2], although we adopt different signs for them.

(2) The Bonnet-Mayers theorem, cf. [Ch, Theorem II.6.1], applies to $S$, and, in particular, $S$ is compact. In fact, the sectional curvatures are the products of two principal curvatures when the hypersurface under consideration is embedded into the Euclidean space, cf. [Ch, Theorem II.2.1], and thus the Ricci curvature is bounded below by a positive constant. See also [Ch, Exercise II.6]. By the same fact we can rewrite (B.2) in terms of the intrinsic sectional curvatures of $S$, assuming the compactness of $S$. We note, however, that for these arguments we need $d \geq 3$ to have nontrivial sectional curvatures.

Proposition B.3. Suppose $\Theta \subset \mathbb{R}^{d}$ is strictly convex. Then the distance function $r(x)=\operatorname{dist}(x, \Theta), x \in \Omega$, satisfies (B.1a) $-(B .1 c)$.

In the sequel we prove Proposition B.3. We first show the convexity of $\Theta$.

Lemma B.4. Let $x \in S$ and set $\tilde{S}_{x}=\exp \left[(T S)_{x}\right]$. Then there exists a neighborhood $U$ of $x$ in $\mathbb{R}^{d}$ such that $\bar{\Theta} \cap \tilde{S}_{x} \cap U=\{x\}$.

This is a sort of local convexity. We omit the proof, just referring to [Ch, Exercise II.4].

Lemma B.5. For any $x, y \in \Theta$ the geodesic $\gamma_{x y}$ connecting $x$ and $y$ lies in $\Theta$.

Proof. Let us assume otherwise, i.e. assume that the set

$$
\Phi=\left\{(x, y) \in \Theta \times \Theta \mid \gamma_{x y}([0,1]) \subset \Theta\right\}
$$

does not coincide with $\Theta \times \Theta$. Since $\gamma_{x y}(t)$ is continuously dependent on $(t, x, y)$, it is clear that $\Phi$ is open in $\Theta \times \Theta$. Choose $\left(x_{0}, y_{0}\right) \in \partial \Phi \subset \Theta \times \Theta$, and then by definition of $\Phi$ we have a proper open subset $\gamma_{x_{0} y_{0}}^{-1}(\Theta) \subsetneq[0,1]$. Since any open subset of $[0,1]$ is a union of at most countable number of open intervals, we can find points $x_{1}, y_{1} \in \gamma_{x_{0} y_{0}}([0,1]) \cap \Theta$ such that the number of component of $\gamma_{x_{1} y_{1}}^{-1}(\Theta)$ is equal to 2. We note we still have $\left(x_{1}, y_{1}\right) \in \partial \Phi$, and hence

$$
\gamma_{x_{1} y_{1}}^{-1}(\Theta)=[0, \tau) \cup(\tau, 1]
$$

for some $\tau \in(0,1)$. In fact, if $\gamma_{x_{1} y_{1}}^{-1}(\Theta)=\left[0, \tau_{1}\right) \cup\left(\tau_{2}, 1\right], 0<\tau_{1}<\tau_{2}<1$, we have to have $\gamma_{x_{1} y_{1}}\left(\left[\tau_{1}, \tau_{2}\right]\right) \subset S$ due to $\left(x_{1}, y_{1}\right) \in \partial \Phi$ and this contradicts Lemma B.4. But (B.3) also contradicts Lemma B.4, and thus $\Phi=\Theta \times \Theta$. 
Now we are ready to give the distorted spherical coordinates for $\Omega$.

Lemma B.6. The exponential map on the outward normal vectors on $S$ :

$$
\exp _{\mid N^{+} S}: N^{+} S \rightarrow \Omega
$$

is bijective.

Proof. We shall intensively use the convexity of $\bar{\Theta}$. Let us denote an element of $N^{+} S$ by $r \nu(\sigma),(r, \sigma) \in(0, \infty) \times S$. If $\exp (r \nu(\sigma)) \in \bar{\Theta}$ for some $(r, \sigma)$, then by the convexity this contradicts the fact that $\nu$ is outward. Thus the image $\exp \left(N^{+} S\right)$ is included in $\Omega$.

Next, assume $\exp (r \nu(\sigma))=\exp \left(r^{\prime} \nu\left(\sigma^{\prime}\right)\right)$ for some $(r, \sigma),\left(r^{\prime}, \sigma^{\prime}\right)$. By the convexity we note that the obstacle $\Theta$ is in one side of the half space devided by the tangent plane at $\sigma$, and by the normality of $\nu(\sigma)$ we obtain $\operatorname{dist}(\exp (r \nu(\sigma)), \Theta)=r$. Thus $r=r^{\prime}$. Moreover, by the convexity of $\bar{\Theta}$ and the minimality of $r=r^{\prime}=$ $\operatorname{dist}(\exp (r \nu(\sigma)), \Theta), \sigma=\sigma^{\prime}$.

Finally take any $x \in \Omega$, and then we can find $y \in \partial \Theta$ such that $\operatorname{dist}(x, \Theta)=$ $\|x-y\|$. Then the geodesic connecting $x$ and $y$ is orthogonal to $\partial \Theta$, because, otherwise, $\|x-y\|$ does not give a minimal distance. This implies $x$ is in the image $\exp \left(N^{+} S\right)$.

As in the proof above we identify $N^{+} S \cong(0, \infty) \times S$ through

$$
N^{+} S \ni r \nu(\sigma) \leftrightarrow(r, \sigma) \in(0, \infty) \times S,
$$

and consider $(r, \sigma) \in(0, \infty) \times S$ as local coordinates of $N^{+} S$. By Lemma B.6 $\exp _{\mid N^{+} S}: N^{+} S \rightarrow \Omega$ is a $C^{\infty}$ bijection, and $r$ is well-defined as the distance function on $\Omega$ : $r(x)=\operatorname{dist}(x, \Theta), x \in \Omega$. The following lemma implies that the pair $(r, \sigma)$ in fact defines local coordinates for $\Omega$.

Lemma B.7. The exponential map $\exp _{\mid N^{+} S}: N^{+} S \rightarrow \Omega$ is a diffeomorphism.

Proof. Parts of the arguments below depend on [Ch, Section III.6]. By Lemma B.6 it suffices to show that $\exp _{\mid N^{+} S}$ is a local diffeomorphism. For $r \geq 0$ and $\sigma \in S$ let $\gamma(\cdot ; r, \sigma)$ be the geodesic defined by

$$
\gamma(t ; r, \sigma)=\exp (\operatorname{tr} \nu(\sigma)), \quad t \in[0,1],
$$

and we consider the vector field $Y_{\alpha}$ along it:

$$
Y_{\alpha}(t)=\partial_{\alpha} \gamma(t ; r, \sigma) ; \quad \partial_{\alpha}=\partial_{\sigma^{\alpha}}, \alpha=2, \ldots, d .
$$

The vector field $Y_{\alpha}$ is so-called the Jacobi field and satisfies the equation

$$
\nabla_{\gamma^{\prime}}^{2} Y_{\alpha}+R\left(\gamma^{\prime}, Y_{\alpha}\right) \gamma^{\prime}=0
$$

with the initial conditions

$$
Y_{\alpha}(0)=\partial_{\alpha}, \quad\left(\nabla_{\gamma^{\prime}} Y_{\alpha}\right)(0)=\pi_{(T S)_{\sigma}}\left(r \nabla_{\alpha} \nu\right)
$$

Let $X_{\alpha}(t)$ be the parallel transport of $\partial_{\alpha} \in(T S)_{\sigma}$ along $\gamma$, i.e.

$$
\left(\nabla_{\gamma^{\prime}} X_{\alpha}\right)(t)=0, \quad X_{\alpha}(0)=\partial_{\alpha},
$$

and seek for a solution to (B.4) and (B.5) of the form $Y_{\alpha}(t)=c_{\alpha}^{\beta}(t) X_{\beta}(t)$. Since $R=0$ and $\left(\nabla_{\alpha} \nu\right)_{\beta}=\left(\left(\nabla^{2} r\right)_{\mid S}\right)_{\alpha \beta}$, we have (B.4) and (B.5) reduced to

$$
\left(c_{\alpha}^{\beta}\right)^{\prime \prime}(t)=0, \quad c_{\alpha}^{\beta}(0)=\delta_{\alpha}^{\beta}, \quad\left(c_{\alpha}^{\beta}\right)^{\prime}(0)=r\left(\left(\nabla^{2} r\right)_{\mid S}\right)_{\alpha \gamma}\left(g_{\mid S}\right)^{\gamma \beta} .
$$


We can solve this as a matrix equation, and hence obtain

$$
Y_{\alpha}(t)=X_{\alpha}(t)+\operatorname{tr}\left(\left(\nabla^{2} r\right)_{\mid S}\right)_{\alpha \gamma}\left(g_{\mid S}\right)^{\gamma \beta} X_{\beta}(t) .
$$

Note that we can choose local coordinates $\sigma$ such that $\partial_{\alpha}, \alpha=2, \ldots, d$, are principal directions of $S$, so that $\left(\nabla^{2} r\right)_{\mid S}$ and $g_{\mid S}$ are written as diagonal matrices. Then by positivity (B.2) it is straightforward to see that $Y_{\alpha}(t), \alpha=2, \ldots, d$, do not degenerate for all $t \geq 0$. Thus $\exp _{\mid N^{+} S}$ is a local diffeomorphism.

Proof of Proposition B.3. By (B.6) we can write the metric of $\Omega$ in terms of coordinates $(r, \sigma)$, and

$$
g=\mathrm{d} r \otimes \mathrm{d} r+\left(g_{\mid S}+r\left(\nabla^{2} r\right)_{\mid S}\right)_{\alpha \gamma}\left(g_{\mid S}\right)^{\gamma \delta}\left(g_{\mid S}+r\left(\nabla^{2} r\right)_{\mid S}\right)_{\delta \beta} \mathrm{d} \sigma^{\alpha} \otimes \mathrm{d} \sigma^{\beta} .
$$

This can be verified by choosing local coordinates $\sigma^{\alpha}$ diagonalizing $\left(\nabla^{2} r\right)_{\mid S}$ and $g_{\mid S}$ and using the fact that the parallel transport does not change the length of vectors. By the representation (B.7) we can compute explicitly

$$
\left(\nabla^{2} r\right)_{\alpha \beta}=\left(\left(\nabla^{2} r\right)_{\mid S}\right)_{\alpha \beta}+r\left(\left(\nabla^{2} r\right)_{\mid S}\right)_{\alpha \gamma}\left(g_{\mid S}\right)^{\gamma \delta}\left(\left(\nabla^{2} r\right)_{\mid S}\right)_{\delta \beta} .
$$

Then for any $c<1$ there exists $r_{0}>0$ such that for all $r \geq r_{0}$

$$
\left(\nabla^{2} r\right)_{\mid S_{r}} \geq c r^{-1} g_{\mid S_{r}} .
$$

Hence we have (B.1b). By (B.7) we can compute $\left|\nabla^{k} r\right|$ in $(r, \sigma)$ coordinates, and then the verification of (B.1c) is also straightforward.

\section{REFERENCES}

[AH] S. Agmon, L. Hörmander: Asymptotic properties of solutions of differential equations with simple characteristics, J. d'Analyse Math., 30 (1976), 1-38.

[BGS] A. Boutet de Monvel, V. Georgescu, A. Soffer, N-body Hamiltonians with hard-core interactions, Rev. Math. Phys. 6 no. 4 (1994), 515-596.

[Ch] I. Chavel, Riemannian geometry. A modern introduction. Second edition. Cambridge Studies in Advanced Mathematics, 98. Cambridge University Press, Cambridge, 2006.

[Da] E.B. Davies, Spectral theory and differential operators, Cambridge, Cambridge University Press 1995.

[De] J. Dereziński, Asymptotic completeness for $N$-particle long-range quantum systems, Ann. of Math. 38 (1993), 427-476.

[DeGé] J. Dereziński, C. Gérard, Scattering theory of classical and quantum N-particle systems, Texts and Monographs in Physics, Berlin, Springer 1997.

[FMS] J. Faupin, J. S. Møller and E. Skibsted, Second order perturbation theory for embedded eigenvalues, Commun. Math. Phys. 306 (2011), 193-228.

[FH] R. Froese, I. Herbst, Exponential bounds and absence of positive eigenvalues for $N$-body Schrödinger operators, Comm. Math. Phys. 87 no. 3 (1982/83), 429-447.

[GY] Y. Gatel, D. Yafaev, On the solutions of the Schrödinger equation with radiation conditions at infinity: the long-range case, Ann. Inst. Fourier, Grenoble 49, no. 5 (1999), 1581-1602.

[Ge] V. Georgescu, On the unique continuation property for Schrödinger Hamiltonians, Helvetica Physica Acta 52 (1979), 655-670.

[GGM] V. Georgescu, C. Gérard and J. S. Møller, Commutators, $C_{0}$-semigroups and resolvent estimates, J. Funct. Anal. 216 (2004), 303-361.

[Gra] G.M. Graf, Asymptotic completeness for $N$-body short-range quantum systems: a new proof, Comm. Math. Phys. 132 (1990), 73-101.

[Gri] M. Griesemer, $N$-body quantum systems with singular potentials, Ann. Inst. Henri Poincaré 69 no. 2 (1998), 135-187.

[Hö] L. Hörmander, The analysis of linear partial differential operators. II-IV, Berlin, Springer 1983-85.

[IS1] K. Ito, E. Skibsted, Scattering theory for Riemannian Laplacians, Preprint 2011. 
[IS2] K. Ito, E. Skibsted, Absence of embedded eigenvalues for Riemannian Laplacians, Preprint 2011.

[Je] A. Jensen, High energy resolvent estimates for Schrödinger operators in Besov spaces, J. Anal. Math. 59 (1992), 45-50.

[JP] A. Jensen, P. Perry, Commutator methods and Besov space estimates for Schrödinger operators, J. Operator Theory 14 (1985), 181-188.

[JK] D. Jerison, C.E. Kenig, Unique continuation and absence of positive eigenvalues for Schrödinger operators, Ann. of Math. (2) 121 no. 3 (1985), 463-494. With an appendix by E. M. Stein.

[Ka] T. Kato, Perturbation theory for linear operators, Springer-Verlag, Berlin, 1995, Reprint of the 1980 edition.

[Mo1] É. Mourre, Absence of singular continuous spectrum for certain selfadjoint operators, Comm. Math. Phys. 78 no. 3 (1980/81), 391-408.

[Mo2] É. Mourre, Operateurs conjugués et propriétés de propagation, Comm. Math. Phys. 91 (1983), 297-300.

[Pe] P. Perry, Exponential bounds and semifiniteness of point spectrum for $N$-body Schrödinger operators, Comm. Math. Phys. 92 (1984), 481-483.

[RS] M. Reed, B. Simon, Methods of modern mathematical physics I-IV, New York, Academic Press 1972-78.

[RT] I. Rodnianski, T. Tao, Effective limiting absorption principles, and applications, Preprint 2011.

[Ro] J. Royer, Limiting absorption principle for the dissipative Helmholtz equation, Comm. PDE. 35 (2010), 1458-1489.

[Sk1] E. Skibsted, Propagation estimates for $N$-body Schrödinger operators, Comm. Math. Phys. 142 (1991), 67-98.

[Sk2] E. Skibsted, Spectral analysis of $N$-body systems coupled to a bosonic field, Rev. Math. Phys. 10 (1998), 989-1026.

[Sk3] E. Skibsted, Sommerfeld radiation condition at threshold, Preprint 2011.

[Vo1] G. Vodev: Uniform estimates of the resolvent of the Laplace-Beltrami operator on infinite volume Riemannian manifolds with cusps, Comm. PDE. 27 no. 7-8 (2002), 1437-1465.

[Vo2] G. Vodev: Local energy decay of solutions to the wave equation for nontrapping metrics, Ark. Mat. 22 (2004), 379-397.

[Wa] X. P. Wang, Microlocal estimates of the stationary Schrödinger equation in semi-classical limit, Partial differential equations and applications, Séminaires et Congrès SMF 15 (2007), 265-308.

[Wo] T. Wolff, Recent work on sharp estimates in second-order elliptic unique continuation problems, J. Geom. Anal. 3 no. 6 (1993), 621-650.

(K. Ito) Graduate School of Pure and Applied Sciences, University of Tsukuba, 1-1-1 Tennodai, Tsukuba Ibaraki, 305-8571 Japan

E-mail address: ito-ken@math.tsukuba.ac.jp

(E. Skibsted) Institut for Matematiske Fag, Aarhus Universitet, Ny Munkegade 8000 Aarhus C, Denmark

E-mail address: skibsted@imf.au.dk 